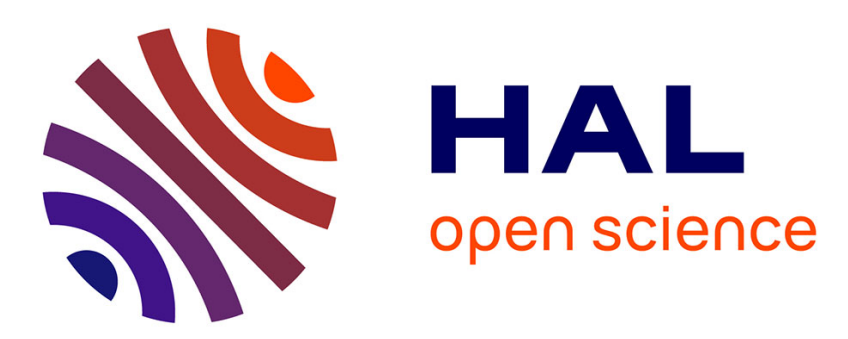

\title{
Perturbation approaches of a planar crack in linear elastic fracture mechanics: A review \\ Veronique Lazarus
}

\section{To cite this version:}

Veronique Lazarus. Perturbation approaches of a planar crack in linear elastic fracture mechanics: A review. Journal of the Mechanics and Physics of Solids, 2011, 59 (2), pp.121-144. 10.1016/j.jmps.2010.12.006 . hal-01904324

\section{HAL Id: hal-01904324 \\ https://hal.science/hal-01904324}

Submitted on 29 Oct 2018

HAL is a multi-disciplinary open access archive for the deposit and dissemination of scientific research documents, whether they are published or not. The documents may come from teaching and research institutions in France or abroad, or from public or private research centers.
L'archive ouverte pluridisciplinaire HAL, est destinée au dépôt et à la diffusion de documents scientifiques de niveau recherche, publiés ou non, émanant des établissements d'enseignement et de recherche français ou étrangers, des laboratoires publics ou privés. 


\title{
Perturbation approaches of a planar crack in Linear Elastic Fracture Mechanics: a review
}

\author{
V. Lazarus \\ UPMC Univ Paris 6, Univ Paris-Sud, CNRS, UMR 7608, Lab FAST, Bat 502, Campus \\ Univ, F-91405, Orsay, France.
}

\begin{abstract}
One current challenge of Linear Elastic Fracture Mechanics (LEFM) is to take into account the non-linearities induced by the crack front deformations. For this, a suitable approach is the crack front perturbation method initiated by Rice (1985). It allows to update the Stress Intensity Factors (SIF) when the crack front of a planar crack is perturbed in its plane. This approach and its later extensions to more complex cases are recalled in this review. Applications concerning the deformation of the crack front when it propagates quasistatically in an homogeneous or heterogeneous media have been considered in brittle fracture, fatigue or subcritical propagation. The crack shapes corresponding to uniform SIF have been derived: cracks with straight or circular fronts, but also when bifurcations exist, with wavy front. For an initial straight crack, it has been shown that, in homogeneous media, in the quasistatic case, perturbations of all lengthscales progressively disappear unless disordered fracture properties yields Family and Vicsek (1985) roughness of the crack front. Extension of those perturbation approaches to more realistic geometries and to coalescence of cracks is also envisaged.
\end{abstract}

Key words: LEFM, brittle fracture, fatigue, crack front deformations, perturbation approach

Consider a crack embedded in an elastic solid that is loaded quasistatically (fig. 1). (i) Under which conditions of loading, (ii) in which direction and (iii) along which distance will this crack propagate? Linear Elastic Fracture

Email address: veronique.lazarus@upmc.fr (V. Lazarus)

$U R L:$ http://www.fast.u-psud.fr/ (V. Lazarus)

Preprint submitted to Journal of the Mechanics and Physics of Solids December 20, 2010 


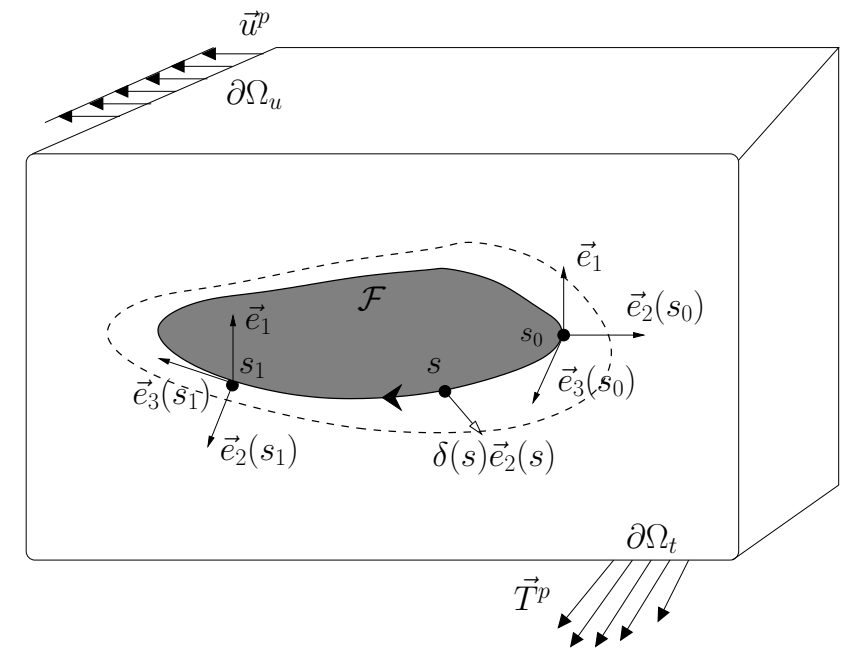

Figure 1: An example of three-dimensional LEFM problem: a three-dimensional planar crack (in grey) embedded in an arbitrary body loaded by external load $\vec{T}^{p}, \vec{u}^{p}$ on $\partial \Omega_{T}$, $\partial \Omega_{u}$. The dashed line corresponds to the position of the initial front $\mathcal{F}$ after an in-plane $\delta(s) \vec{e}_{2}(s)$ advance.

Mechanics (LEFM) aims at answering these questions. It is widely applied in several fields, for instance: in engineering applications (Anderson, 1991) for obvious safety reasons; in geological applications (Aydin and Pollard, 1988; Atkinson, 1987) as earthquakes (Liu and Rice, 2005; Fisher et al., 1997; Grob et al., 2009; Bonamy, 2009), basalt columns (Goehring et al., 2009) or in the soft matter domain (Gauthier et al., 2010). The principal aim of this review, in addition to a brief overview of the answers to the first two questions, is linked to the third question and concerns the crack front deformation of a three-dimensional planar crack during its propagation.

Nowadays, several different approaches are developed within LEFM. Traditional approach, in the continuity of the pioneer works of Griffith (1920) and Irwin (1958), is based on the local mechanical and energetic fields near the crack front and uses propagation criterions based on the Stress Intensity Factors $K_{1}, K_{2}, K_{3}$ or the elastic energy release rate $\mathcal{G}$. This approach aims at predicting the propagation conditions (loading, path) of a preexisting crack, but is unable to deal with the crack initiation problem.

Recently new approaches have been developed that are able, in theory, 
to deal with both the crack initiation and propagation paths: the energetic variational minimisation approach to fracture and the phase-field method. The first (i) has been shown to include the traditional approach (Francfort and Marigo, 1998; Bourdin et al., 2008), (ii) has been approximated for numerical purposes by non-local damage first-gradient model (Bourdin et al., 2000) and (iii) has been applied to several brittle fracture problems as stability problems (Benallal and Marigo, 2007), the deterioration of the French Panthéon (Lancioni and Royer-Carfagni, 2009), the propagation direction in presence of mode 2 (Chambolle et al., 2009), crack patterns in shrinkage, drying or cooling, problems (Lazarus et al., 2009). The second, first developed for solidification front (Caginalp and Fife, 1986; Collins and Levine, 1985) was further extended to brittle fracture (Karma et al., 2001) and has shown their efficiency to resolve problems of path determination in 2D (Henry and Levine, 2004; Hakim and Karma, 2009; Corson et al., 2009) and 3D (Pons and Karma, 2010).

But the traditional approach has still its place thanks to the maturity acquired by its longer history: in particular, to deal with the crack front shape deformations an efficient perturbation method of the crack front, pioneered by Rice (1985), has been used. The method allows to update the stress intensity factors (that are prerequisite to any crack propagation prediction) for any small perturbation of the crack front without resolving the whole elasticity problem. On the one hand, the initial method of Rice (1985) has been applied to the propagation of cracks in disordered heterogeneous by the statistical physics community. On the other, it has be extended and applied to more and more complex problems by the fracture mechanics community. It has recently gained a renewal of interest (Dalmas et al., 2009) with the creation of new intelligent materials by deposition of a stack of nanometric thin layers having specific functions (as for instance, optical, thermal, selfcleaning) that may perturb locally the crack front.

A review concerning the roughening of the front in disordered heterogeneous materials has recently be done by Bonamy (2009) from a statistical physics point of view. Here, the aim is to do a review of the crack front perturbation approaches from a mechanical point of view. It is focused, as in Bonamy (2009), mainly on the slow crack growth regime in which the crack speed is negligeable in comparison to Rayleigh speed. The high speed regime is beyond the scope of this paper although at some places some key references are given.

The outline of the paper is as follows. After a brief overview of the 
traditional LEFM approach (section 1), the perturbation method is presented in the general case (section 2) and then for some model selected problems (section 3). Application to crack propagation in homogeneous media (section 4 ), crack trapping by tougher obstacles (section 5), propagation in disordered media (section 6) are then developed.

\section{Overview of the traditional LEFM approach}

\subsection{Definition of the SIFs}

Consider a crack embedded in a linear elastic body. Suppose that the evolution is quasistatic ${ }^{1}$. Let $\mathcal{F}$ denote the crack front and $s$ some curvilinear abscissa along it. The front is supposed to be smooth so that at each point $s$ of $\mathcal{F}$, one can define a local basis of vectors $\left(\vec{e}_{1}(s), \vec{e}_{2}(s), \vec{e}_{3}(s)\right)$ in the following way :

1. $\vec{e}_{3}(s)$ is tangent to $\mathcal{F}$ and oriented in the same direction as the curvilinear abscissa $s$;

2. $\vec{e}_{2}(s)$ is in the crack plane, orthogonal to $\mathcal{F}$ and oriented in the direction of propagation;

3. $\vec{e}_{1}(s) \equiv \vec{e}_{1}$ is orthogonal to the crack plane and oriented in such a way that the basis $\left(\vec{e}_{2}(s), \vec{e}_{1}, \vec{e}_{3}(s)\right)$ is direct $^{2}$.

The $\operatorname{SIFs} K_{j}(s), j=1,2,3$ at point $s$ are then defined by the following formula, where Einstein's summation convention is employed :

$$
\lim _{r \rightarrow 0} \sqrt{\frac{2 \pi}{r}} \llbracket \vec{u}(s, r) \rrbracket \equiv 8 \Lambda_{i j} K_{j}(s) \vec{e}_{i}(s)
$$

In this expression $\llbracket \vec{u}(s, r) \rrbracket$ denotes the displacement discontinuity across the crack plane, oriented by the vector $\vec{e}_{1}$, at the distance $r$ behind the point $s$ of $\mathcal{F}$, in the direction of the vector $-\vec{e}_{2}(s)$. Also, $\left(\Lambda_{i j}\right)_{1 \leq i \leq 3,1 \leq j \leq 3} \equiv \boldsymbol{\Lambda}$ is the

\footnotetext{
${ }^{1}$ In the dynamic case, one may refer e.g. to Freund (1972b, 1973, 1974), Kostrov (1975) for pioneer works and Ravi-Chandar (1998), Fineberg and Marder (1999), Freund (2000), Bouchbinder et al. (2010) for more recent publications.

${ }^{2}$ the order of the vectors may seem a little surprising but this definition of the local basis presents the advantage that the mode 1, (resp. 2 and 3) corresponds to the displacement jump along the vector with the same numbering, that is $\vec{e}_{1}$ (resp. $\vec{e}_{2}$ and $\vec{e}_{3}$ )
} 
diagonal matrix defined by

$$
\Lambda \equiv \frac{1}{E}\left(\begin{array}{ccc}
1-\nu^{2} & 0 & 0 \\
0 & 1-\nu^{2} & 0 \\
0 & 0 & 1+\nu
\end{array}\right)
$$

\subsubsection{Brittle fracture}

Concerning brittle fracture, Griffith (1920)'s criterion is extensively used: it states that the crack propagation occurs if the elastic energy released by the crack propagation $\mathcal{G}$ is sufficient to counterbalance the fracture energy $\Gamma$ necessary to create new surfaces:

$$
\begin{aligned}
& \mathcal{G}<\Gamma \Rightarrow \text { no propagation, } \\
& \mathcal{G}=\Gamma \Rightarrow \text { possible propagation. }
\end{aligned}
$$

SIFs and energy release rate $\mathcal{G}$ are linked by Irwin (1957)'s formula (Einstein summation convention is used):

$$
\mathcal{G}=K_{i} \Lambda_{i j} K_{j}
$$

so that, in mode $1\left(K_{2}=0, K_{3}=0\right)$, Griffith (1920)'s criterion is equivalent to Irwin (1958)'s criterion which states that the crack propagates if the Stress Intensity Factor $K_{1}$ at the crack tip exceeds the local toughness $K_{c}$ :

$$
\begin{array}{r}
K_{1}<K_{c} \Rightarrow \text { no propagation, } \\
K_{1}=K_{c} \Rightarrow \text { possible propagation. }
\end{array}
$$

\footnotetext{
${ }^{3}$ A similar formula holds for an arbitrary anisotropic medium but the matrix $\boldsymbol{\Lambda}$ is then no longer diagonal (Broberg, 1999, §4.14), and also for the dynamic case, but then $\boldsymbol{\Lambda}$ depends on the crack velocity (Freund, 2000, §5.3).
} 
Above the threshold, Griffith's criterion remains valid (Sharon and Fineberg, 1999) provided that the velocity dependance of $\boldsymbol{\Lambda}$ is taken into account (Freund, 2000) in equation (2). In particular, in mode 1 and in the slow growth regim $\left(v \ll C_{R}\right)$, it gives for the crack velocity $v$ :

$$
v=\frac{2 C_{R}}{K_{c}}\left(K_{1}-K_{c}\right) \text { for } K_{1} \geq K_{c}
$$

$C_{R}$ being the Rayleigh speed.

\subsubsection{Subcritical or fatigue propagation}

Concerning the subcritical propagation below the brittle fracture threshold $\left(K_{c}\right.$ or $\left.\Gamma\right)$, Paris' law (Paris et al., 1961; Erdogan and Paris, 1963) with a threshold $\mathcal{G}_{0}$ or without $\left(\mathcal{G}_{0}=0\right)$ is often used. It states that the crack velocity $v$ goes as a power-law with the excess energy release rate $\mathcal{G}$ :

$$
v=C\left(\mathcal{G}-\mathcal{G}_{0}\right)^{\beta},
$$

In the case of mode 1 with $\mathcal{G}_{0}=0$, it is equivalent to (use (5) and (2)):

$$
v=C^{\prime} K_{1}^{N} \text { with } N=2 \beta \text { and } C^{\prime}=C\left(\frac{1-\nu^{2}}{E}\right)^{\beta}
$$

For fatigue, the same expressions (9) and (10) are valid if $v$ is re-interpreted as advance during one cycle and $\mathcal{G}$ as variation of the energy release rate during one cycle. An overview of the values of $\beta$ or $N$ and $C$ for engineering materials can be found in Fleck et al. (1994). It's physical background (Vieira et al., 2008) and its validity field (Ciavarella et al., 2008) are still the subject of many research papers.

\subsection{Crack propagation direction criterions}

In homogeneous isotropic elastic media, except in some special conditions, it is well known that whatever the external loading, the crack front bifurcates in order to reach a situation of pure tension loading as the crack propagates (Hull, 1993). Hence, planar crack propagation is generally stable under mode 1 loading and unstable under mode 2 or 3 . A literature survey of mixed mode crack growth can be found in Qian and Fatemi (1996). Under mode $(1+2)$ conditions, the crack kinks to make mode 2 vanish. The value 
of the corresponding kink angle can be obtained, for instance, by the Principle of Local Symmetry (PLS) of Goldstein and Salganik (1974) or by the maximum tangential stress criterion (MTS; Erdogan and Sih (1963)). The difference between these two criterions has been discussed by Amestoy and Leblond (1992). In presence of mode 3, the problem becomes threedimensional and seldom papers deal with the prediction of the propagation path in this condition. Among them, Lazarus and Leblond (1998a), Lazarus et al. (2001b), Lazarus et al. (2008) consider the particular case of 3 or 4 point bending experiments and Cooke and Pollard (1996), Lin et al. (2010), Pons and Karma (2010) the segmentation of the front. Theoretical (Karma et al., 2011) and experimental (Lazarus et al., 2011a) papers on segmentation and coarsening are also currently under progress.

In some particular situations, even in presence of mode 2 or 3 , planar crack propagation may be stable. It is the case for instance when the crack is channelled along a planar surface of low fracture resistance, which can be the case for instance along a geological fault or in composite materials. It may also be the case in fatigue due to the presence of friction (Doquet and Bertolino, 2008).

\subsection{Crack perturbation approaches}

To predict the propagation path applying crack advance and propagation criterions described above, perturbation methods have been used. They are based on the pioneer works of Bueckner (1970), Rice $(1972,1985)$. Their method has been extended in both the static and dynamic case.

Nevertheless, in this review we focus further on the static case ${ }^{4}$. A first set of papers considers the out-of-plane perturbation of the faces of a planar crack: the first order variation of the SIF are given in Movchan et al. (1998). It is applied by Obrezanova et al. (2002b) to generalise the Cotterell and Rice (1980)'s 2D stability analysis of a crack to small out-of-plane deviation of its path. We are currently extending this approach to 3D out of plane instabilities in mixed mode $1+3$ (Leblond et al., 2011). A second set of papers gives the expressions of the SIFs along the front of an arbitrary kinked and curved infinitesimal extension of some arbitrary crack: Leblond (1989) and Amestoy and Leblond (1992) in 2D, and Leblond (1999) and Leblond et al.

\footnotetext{
${ }^{4}$ In the dynamic fracture case some key references are Rice et al. (1994), Willis and Movchan (1995), Willis and Movchan (1997), Woolfries and Willis (1999), Obrezanova et al. (2002a).
} 
(1999) in 3D. These expressions have been applied for instance, to show that the PLS and the MTS yield very close but distinct kink angles (Amestoy and Leblond, 1992) or to the crack front rotation and segmentation in mixed mode $1+3$ or $1+2+3$ (Lazarus and Leblond, 1998a; Lazarus et al., 2001a,b). A third set of papers consider the same problem then the second in the particular case of a planar crack with a coplanar extension. They are the main object of this review and are developed below.

\section{In-plane crack front perturbation approaches for an arbitrary planar crack}

Consider a plane crack of arbitrary shape embedded in some isotropic static elastic medium subjected to some arbitrary loading: given forces $\vec{T}^{p}$ along $\partial \Omega_{T}$ and given displacements $\vec{u}^{p}$ along $\partial \Omega_{u}$ (Figure 1 ). The aim of this section is to give the first order variation of the stress intensity factors due to small in-plane perturbation of the crack front. Such formulae have first been derived in several particular cases notably by Rice and coworkers, and then generalised to more arbitrary problems. Here the historical chronology is not respected: first, in the present section, the most general formulae are recalled by relying on the paper of Favier et al. (2006a). They are then particularised to some model problems in section 3.

\subsection{Definitions and elementary properties of weight functions}

Definitions. Let $k_{i j}\left(\mathcal{F} ; s^{\prime} ; s, r\right)$ denote the $i$-th SIF at the point $s^{\prime}$ of the crack front $\mathcal{F}$ resulting from application of a pair of opposite unit point forces equal to $\pm \vec{e}_{j}(s)$ on the upper $(+)$ and lower $(-)$ crack surfaces at a distance $r$ behind the point $s$ of the crack front the other loading being supposed to be zero $\left(\vec{T}^{p}=\overrightarrow{0}\right.$ along $\partial \Omega_{T}$ and $\vec{u}^{p}=\overrightarrow{0}$ along $\left.\partial \Omega_{u}\right)$. These nine scalar functions are called the crack face weight functions (CFWFs).

The functions $k_{i j}\left(\mathcal{F} ; s^{\prime} ; s, r\right) / \sqrt{r}$ are known to have a well-defined limit for $r \rightarrow 0$ (see for instance Leblond et al. (1999)). We then define the matrix $\left(W_{i j}\left(s^{\prime}, s\right)\right)_{1 \leq i \leq 3,1 \leq j \leq 3} \equiv \mathbf{W}\left(s^{\prime}, s\right)$ by the formula

$$
W_{i j}\left(s^{\prime}, s\right) \equiv \pi \sqrt{\frac{\pi}{2}} D^{2}\left(s, s^{\prime}\right) \lim _{r \rightarrow 0} \frac{k_{i j}\left(\mathcal{F} ; s^{\prime} ; s, r\right)}{\sqrt{r}}
$$

where $D\left(s, s^{\prime}\right)$ denotes the cartesian distance between points $s$ and $s^{\prime}$. The functions $W_{i j}\left(s^{\prime}, s\right)$ in fact depend on the crack front shape, just like the 
Properties. The CFWFs are positively homogeneous of degree $-3 / 2$; that is, if all distances are multiplied by some positive factor $\lambda$, the CFWFs are multiplied by $\lambda^{-3 / 2}$. The definition (11) of the functions $W_{i j}\left(s^{\prime}, s\right)$ then implies that they are positively homogeneous of degree 0 :

$$
\mathbf{W}\left(\lambda s^{\prime}, \lambda s\right)=\mathbf{W}\left(s^{\prime}, s\right) \quad \forall \lambda>0
$$

Since tensile and shear problems are uncoupled for a planar crack in an infinite body, whatever the shape of the crack front, the components $k_{12}, k_{13}$, $k_{21}$ and $k_{31}$ of the CFWFs are zero, so that by equation (11),

$$
W_{12}\left(s^{\prime}, s\right) \equiv W_{13}\left(s^{\prime}, s\right) \equiv W_{21}\left(s^{\prime}, s\right) \equiv W_{31}\left(s^{\prime}, s\right) \equiv 0 .
$$

Considering two problems, one with point forces equal to $\pm \vec{e}_{i}$ exerted on the crack faces at $(s, r)$ and one with point forces equal to $\pm \vec{e}_{j}$ exerted on the crack faces at $\left(s^{\prime}, r^{\prime}\right)$, applying Betti's theorem, and using equations (1) and (11), one sees that the kernels obey the following "symmetry" property :

$$
\Lambda_{i m} W_{m j}\left(s, s^{\prime}\right)=\Lambda_{j m} W_{m i}\left(s^{\prime}, s\right) .
$$

Finally, Leblond et al. (1999) have shown that the limit of $\mathbf{W}\left(s, s^{\prime}\right)$ when $s^{\prime} \rightarrow s$ is universal, i.e. that it does not depend on the geometry. It is linked to the behaviour of the weight-functions when the point of application of the loading tends toward the point of observation of the SIF which is a local property independent of the far geometry. The values of this limit are:

$$
\left\{\begin{array}{l}
\lim _{s^{\prime} \rightarrow s} W_{11}\left(s, s^{\prime}\right)=1 \\
\lim _{s^{\prime} \rightarrow s} W_{22}\left(s, s^{\prime}\right)=\frac{2-3 \nu}{2-\nu} \\
\lim _{s^{\prime} \rightarrow s} W_{33}\left(s, s^{\prime}\right)=\frac{2+\nu}{2-\nu} \\
\lim _{s^{\prime} \rightarrow s} W_{23}\left(s, s^{\prime}\right)=0
\end{array}\right.
$$




\subsection{First order variation of the stress intensity factors}

Let us now assume that the crack advances, under constant loading, by a small distance $\delta(s)$ within its plane in the direction perpendicular to its front (fig. 1). It has been shown in Favier et al. (2006a) that, for any loading, if $\delta\left(s_{0}\right)=0$,

$$
\delta \mathbf{K}\left(s_{0}\right)=\mathbf{N} \cdot \mathbf{K}\left(s_{0}\right) \delta^{\prime}\left(s_{0}\right)+\frac{1}{2 \pi} \mathrm{PV} \int_{\mathcal{F}} \frac{\mathbf{W}\left(s_{0}, s\right)}{D^{2}\left(s_{0}, s\right)} \cdot \mathbf{K}(s) \delta(s) \mathrm{d} s .
$$

The condition $\delta\left(s_{0}\right)=0$ ensures the existence of the Principal Value integral $\mathrm{PV} \int$. The quantities $\mathbf{K}(s) \equiv\left(K_{i}(s)\right)_{1 \leq i \leq 3}$ and $\delta \mathbf{K}(s) \equiv\left(\delta K_{i}(s)\right)_{1 \leq i \leq 3}$ here are the column vectors of initial SIFs and variations of these SIFs, and $\mathbf{N} \equiv\left(N_{i j}\right)_{1 \leq i \leq 3,1 \leq j \leq 3}$ is the matrix defined by

$$
\mathbf{N} \equiv \frac{2}{2-\nu}\left(\begin{array}{ccc}
0 & 0 & 0 \\
0 & 0 & -1 \\
0 & 1-\nu & 0
\end{array}\right)
$$

Equation (16) is identical to Leblond et al. (1999)'s general equation (30) (with the notation $\frac{1}{2 \pi} \frac{\mathbf{W}\left(s_{0}, s\right)}{D^{2}\left(s_{0}, s_{1}\right)}$ instead of $\mathbf{Z}\left(s_{0}, s\right)$ ), in the special case of a planar crack with coplanar extension (and zero crack advance at the point $\left.s_{0}\right)$. It shall be noticed that the variation of the SIFs at a particular point $s_{0}$ depends in an non-local manner on the crack perturbation along all the front. It is due to long-range elastic interactions.

The restriction $\delta\left(s_{0}\right)=0$ will now be removed by two methods:

1. Using a trick of Rice (1989), that consists of decomposing an arbitrary motion of the crack front defined by the normal advance $\delta(s)$ into two steps :

(a) A translatory motion of displacement vector $\delta\left(s_{0}\right) \vec{e}_{2}\left(s_{0}\right)$. This motion brings the point $s_{0}$ to its correct final position while leaving the crack front shape unchanged. The corresponding normal advance $\delta_{*}(s)$ is given, to first order in $\delta(s)$, by

$$
\delta_{*}(s)=\delta\left(s_{0}\right) \vec{e}_{2}\left(s_{0}\right) \cdot \vec{e}_{2}(s) .
$$

The associated variation of $\mathbf{K}(s)$ will be denoted $\delta_{*} \mathbf{K}(s)$. 
(b) A motion with normal advance given by $\delta(s)-\delta_{*}(s)$. This advance is zero at point $s_{0}$ so that the corresponding variation of $\mathbf{K}\left(s_{0}\right)$ is given by equation $(16)$, with $\delta^{\prime}\left(s_{0}\right)-\delta_{*}^{\prime}\left(s_{0}\right)=\delta^{\prime}\left(s_{0}\right)$ since $\delta_{*}^{\prime}\left(s_{0}\right)=$ 0 by equation (18).

Adding up the contributions from these two motions, one gets the final expression of the variation of the SIFs under constant loading in the general case:

$$
\begin{aligned}
\delta \mathbf{K}\left(s_{0}\right)= & \delta_{*} \mathbf{K}\left(s_{0}\right)+\mathbf{N} \cdot \mathbf{K}\left(\mathbf{s}_{\mathbf{0}}\right) \delta^{\prime}\left(s_{0}\right) \\
& +\frac{1}{2 \pi} \mathrm{PV} \int_{\mathcal{F}} \frac{\mathbf{W}\left(s_{0}, s\right)}{D^{2}\left(s_{0}, s\right)} \cdot \mathbf{K}(s)\left[\delta(s)-\delta_{*}(s)\right] \mathrm{d} s .
\end{aligned}
$$

This expression allows to update the SIFs knowing the initial SIFs, FK and the displacement provided that the quantity $\delta_{*} \mathbf{K}$ can be calculated. The unknown quantity $\delta_{*} \mathbf{K}\left(s_{0}\right)$ is equal to zero if the translatory motion $\delta\left(s_{0}\right) \vec{e}_{2}\left(s_{0}\right)$ leaves the problem unchanged. It is for instance the case if the crack front is far from any boundary so that the media can be assumed to be infinite submitted to remote stress loading. Then, the first order formula simply becomes:

$$
\begin{aligned}
\delta \mathbf{K}\left(s_{0}\right)= & \mathbf{N} \cdot \mathbf{K}\left(\mathbf{s}_{\mathbf{0}}\right) \delta^{\prime}\left(s_{0}\right) \\
& +\frac{1}{2 \pi} \mathrm{PV} \int_{\mathcal{F}} \frac{\mathbf{W}\left(s_{0}, s\right)}{D^{2}\left(s_{0}, s\right)} \cdot \mathbf{K}(s)\left[\delta(s)-\delta\left(s_{0}\right) \vec{e}_{2}\left(s_{0}\right) \cdot \vec{e}_{2}(s)\right] \mathrm{d} s .
\end{aligned}
$$

2. Another possibility is to proceed as Leblond et al. (1999) and to decompose the normal advance $\delta(s)$ into a uniform advance $\delta\left(s_{0}\right)$ (denote $\left[\delta \mathbf{K}\left(s_{0}\right)\right]_{\delta(s) \equiv \delta\left(s_{0}\right)}$ the corresponding first order variation of the SIFs) and the advance $\delta(s)-\delta\left(s_{0}\right)$ for which the equation (16) can be used. The final expression then reads:

$$
\begin{aligned}
\delta \mathbf{K}\left(s_{0}\right)= & {\left[\delta \mathbf{K}\left(s_{0}\right)\right]_{\delta(s) \equiv \delta\left(s_{0}\right)}+\mathbf{N} \cdot \mathbf{K}\left(\mathbf{s}_{\mathbf{0}}\right) \delta^{\prime}\left(s_{0}\right) } \\
& +\frac{1}{2 \pi} \mathrm{PV} \int_{\mathcal{F}} \frac{\mathbf{W}\left(s_{0}, s\right)}{D^{2}\left(s_{0}, s\right)} \cdot \mathbf{K}(s)\left[\delta(s)-\delta\left(s_{0}\right)\right] \mathrm{d} s .
\end{aligned}
$$

This expression is useful if one can calculate $\left[\delta \mathbf{K}\left(s_{0}\right)\right]_{\delta(s) \equiv \delta\left(s_{0}\right)}$. It is the case for instance if the uniform advance $\delta(s) \equiv \delta\left(s_{0}\right)$ doesn't change the geometry of the problem as for a circular, straight half-plane or tunnel crack. 
Formula (16) and its corollaries (19), (20), (21) have been derived for homogeneous isotropic elastic solids. For cracks at the interface between two elastic solids, such a formula exists in the sole case of a half-plane crack: the first order variation of the SIFs can be found in Lazarus and Leblond (1998b) and using an other formalism (Wiener-Hopf analysis) in Bercial-Velez et al. (2005), the connection between the two methods having been done by Piccolroaz et al. (2007).

\subsection{First order variation of the fundamental kernel}

To derive higher order variation of the SIFs, the first order variation of the fundamental kernel is necessary. It has been shown by Rice (1989) in mode 1 and Favier et al. (2006a) in modes 2+3 that:

$$
\begin{aligned}
\delta \mathbf{W}\left(s_{0}, s_{1}\right)= & \mathbf{N} \cdot \mathbf{W}\left(s_{0}, s_{1}\right) \delta^{\prime}\left(s_{0}\right)-\mathbf{W}\left(s_{0}, s_{1}\right) \cdot \mathbf{N} \delta^{\prime}\left(s_{1}\right) \\
& +\frac{D^{2}\left(s_{0}, s_{1}\right)}{2 \pi} \mathrm{PV} \int_{\mathcal{F}} \frac{\mathbf{W}\left(s_{0}, s\right) \cdot \mathbf{W}\left(s, s_{1}\right)}{D^{2}\left(s_{0}, s\right) D^{2}\left(s_{1}, s\right)} \delta(s) \mathrm{d} s,
\end{aligned}
$$

if $\delta\left(s_{0}\right)=\delta\left(s_{1}\right)=0$. In order to get rid of these conditions, one must imagine a motion $\delta_{* *}(s)$ such as $\delta_{* *}\left(s_{0}\right)=\delta\left(s_{0}\right)$ and $\delta_{* *}\left(s_{1}\right)=\delta\left(s_{1}\right)$. Denote $\delta_{* *} \mathbf{W}\left(s_{0}, s_{1}\right)$ the corresponding variation of the kernel. Equation (22) then becomes:

$$
\begin{aligned}
\delta \mathbf{W}\left(s_{0}, s_{1}\right) & =\delta_{* *} \mathbf{W}\left(s_{0}, s_{1}\right)+\mathbf{N} \cdot \mathbf{W}\left(\mathbf{s}_{0}, \mathbf{s}_{1}\right)\left[\delta^{\prime}\left(s_{0}\right)-\delta_{* *}^{\prime}\left(s_{0}\right)\right] \\
& -\mathbf{W}\left(s_{0}, s_{1}\right) \cdot \mathbf{N}\left[\delta^{\prime}\left(s_{1}\right)-\delta_{* *}^{\prime}\left(s_{1}\right)\right] \\
& +\frac{D^{2}\left(s_{0}, s_{1}\right)}{2 \pi} \mathrm{PV} \int_{\mathcal{F}} \frac{\mathbf{W}\left(s_{0}, s\right) \cdot \mathbf{W}\left(s, s_{1}\right)}{D^{2}\left(s_{0}, s\right) D^{2}\left(s_{1}, s\right)}\left[\delta(s)-\delta_{* *}(s)\right] \mathrm{d} s .
\end{aligned}
$$

A difficulty is to be able to define $\delta_{* *}(s)$ such as $\delta_{* *} W\left(s_{0}, s_{1}\right)$ can be calculated. This problem has not been solved at present in the general case. In the particular case of an infinite body subjected to uniform remote loading, one can always find a combination of a translatory motion, a rotation and a homothetical transformation bringing two distinct points $s_{0}, s_{1}$ from any initial positions to any final positions (This is obvious using a complex variable formalism and noting that such transformations are of the form $f(z)=a z+b$ where $a$ and $b$ are arbitrary complex parameters). Such a combination leaves 
the kernels unaffected so that $\delta_{* *} W\left(s_{0}, s_{1}\right)=0$. Equation (23) then yields:

$$
\begin{aligned}
\delta \mathbf{W}\left(s_{0}, s_{1}\right)= & \mathbf{N} \cdot \mathbf{W}\left(\mathbf{s}_{\mathbf{0}}, \mathbf{s}_{\mathbf{1}}\right)\left[\delta^{\prime}\left(s_{0}\right)-\delta_{* *}^{\prime}\left(s_{0}\right)\right] \\
& -\mathbf{W}\left(s_{0}, s_{1}\right) \cdot \mathbf{N}\left[\delta^{\prime}\left(s_{1}\right)-\delta_{* *}^{\prime}\left(s_{1}\right)\right] \\
& +\frac{D^{2}\left(s_{0}, s_{1}\right)}{2 \pi} \mathrm{PV} \int_{\mathcal{F}} \frac{\mathbf{W}\left(s_{0}, s\right) \cdot \mathbf{W}\left(s, s_{1}\right)}{D^{2}\left(s_{0}, s\right) D^{2}\left(s_{1}, s\right)}\left[\delta(s)-\delta_{* *}(s)\right] \mathrm{d} s .
\end{aligned}
$$

\subsubsection{Circular cracks}

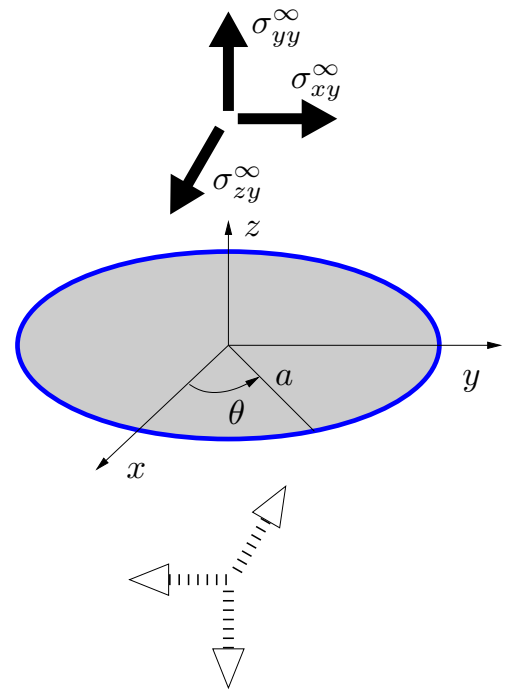

(a) An internal circular crack in an infinite media loaded by remote tensile and shear stresses

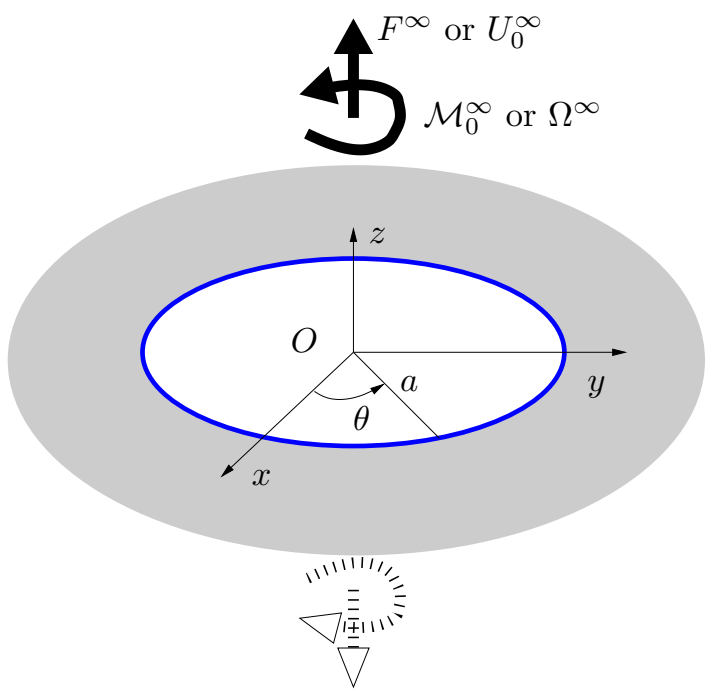

(b) An external circular crack in an infinite media loaded by remote (i) tensile net centred force or displacement and (ii) momentum or rotation

Figure 2: Several problems involving a circular crack.

Two cases are considered, an internal and an external crack: 
- For the internal circular crack such as $\partial \Omega_{u}=\varnothing$, for instance loaded by remote stresses (fig. 2(a)), the value of the non-zero components of the kernel W are (Kassir and Sih, 1975; Tada et al., 1973; Bueckner, 1987; Gao and Rice, 1987b; Gao, 1988):

$$
\left\{\begin{array}{l}
W_{11}\left(\theta_{0}, \theta_{1}\right)=1 \\
W_{22}\left(\theta_{0}, \theta_{1}\right)=\frac{2 \cos \left(\theta_{0}-\theta_{1}\right)-3 \nu}{2-\nu} \\
W_{33}\left(\theta_{0}, \theta_{1}\right)=\frac{2(1-\nu) \cos \left(\theta_{0}-\theta_{1}\right)+3 \nu}{2-\nu} \\
W_{23}\left(\theta_{0}, \theta_{1}\right)=\frac{1}{1-\nu} W_{32}\left(\theta_{1}, \theta_{0}\right)=\frac{2 \sin \left(\theta_{0}-\theta_{1}\right)}{2-\nu}
\end{array}\right.
$$

- For the external circular crack (fig. 2(b)), only the value in mode 1 is known (Stallybrass, 1981; Gao and Rice, 1987a; Rice, 1989) for several cases of remote boundary conditions:

- when remote points are clamped (given $U_{0}^{\infty}=0, \Omega^{\infty}=0$ ):

$$
W_{11}\left(\theta_{0}, \theta_{1}\right)=1
$$

- when remote points can not rotate but can move in the $\vec{e}_{1}$ direction (given $F^{\infty}=0, \Omega^{\infty}=0$ ):

$$
W_{11}\left(\theta_{0}, \theta_{1}\right)=1+4 \sin ^{2}\left(\frac{\theta_{0}-\theta_{1}}{2}\right)
$$

- when remote points can not move in the $\vec{e}_{1}$ direction, but can rotate (given $U_{0}^{\infty}=0, \mathcal{M}_{0}^{\infty}=0$ ):

$$
W_{11}\left(\theta_{0}, \theta_{1}\right)=1+24 \sin ^{2}\left(\frac{\theta_{0}-\theta_{1}}{2}\right) \cos \left(\theta_{0}-\theta_{1}\right)
$$

- when remote points are constrained against any motion (given $\left.F^{\infty}=0, \mathcal{M}_{0}^{\infty}=0\right)$ :

$$
W_{11}\left(\theta_{0}, \theta_{1}\right)=1+4 \sin ^{2}\left(\frac{\theta_{0}-\theta_{1}}{2}\right)\left[1+6 \cos \left(\theta_{0}-\theta_{1}\right)\right]
$$




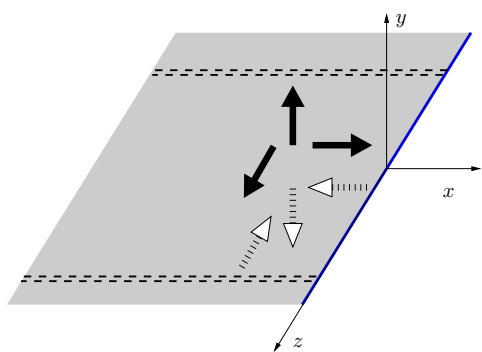

(a) A half-plane crack in an infinite media loaded by remote tensile and shear stresses

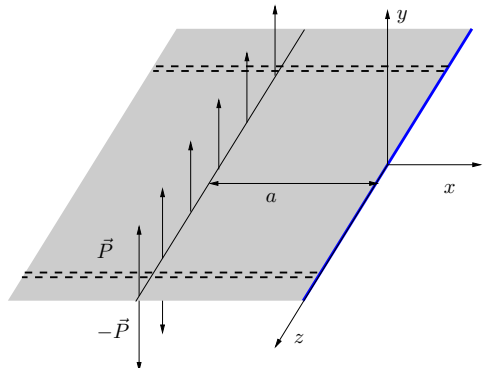

(b) A half-plane crack with uniform line tractions

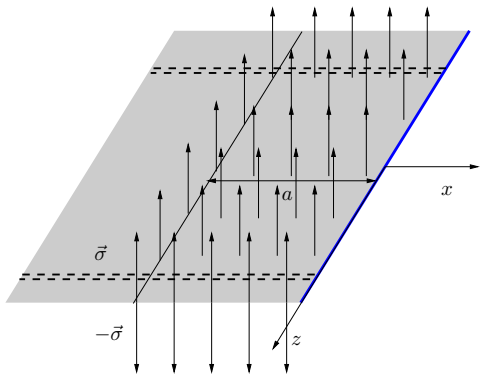

(c) A half-plane crack with uniform surface tractions

Figure 3: Several problems involving a half-plane crack

\subsubsection{Half-plane crack}

In the case of a half-plane crack with $\partial \Omega_{u}=\varnothing$, loaded for instance by remote stresses (fig. 3(a)) or line (fig. 3(b)) or surface traction (fig. 3(c)), the kernel is (Meade and Keer, 1984; Bueckner, 1987; Rice, 1985; Gao and Rice, 1986):

$$
\left\{\begin{array}{l}
W_{11}\left(z_{1}, z_{0}\right)=1 \\
W_{22}\left(z_{1}, z_{0}\right)=\frac{2-3 \nu}{2-\nu} \\
W_{33}\left(z_{1}, z_{0}\right)=\frac{2+\nu}{2-\nu} \\
W_{23}\left(z_{1}, z_{0}\right)=0
\end{array}\right.
$$

\subsubsection{Tunnel-cracks}

The model of half-plane crack is widely used due to its simplicity, but it lacks a lengthscale. To introduce a lengthscale, Leblond and coauthors have 


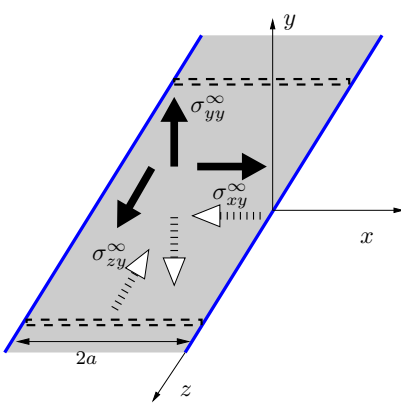

(a) A tunnel-crack in an infinite media loaded by remote tensile and shear stresses

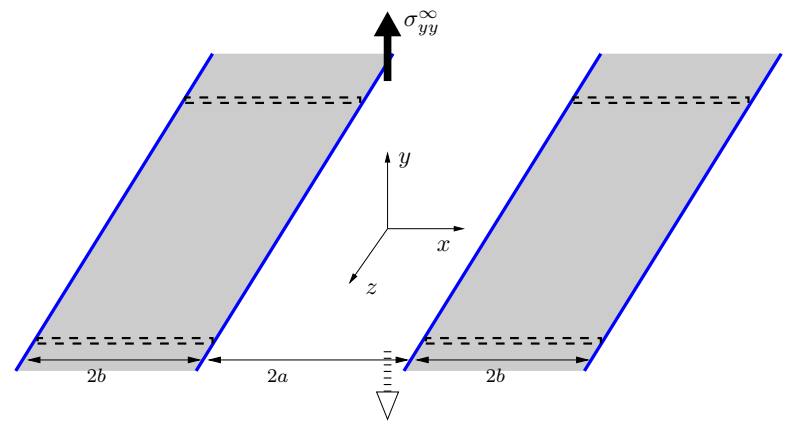

(b) Two tunnel-cracks, in an infinite media loaded by remote tensile stresses

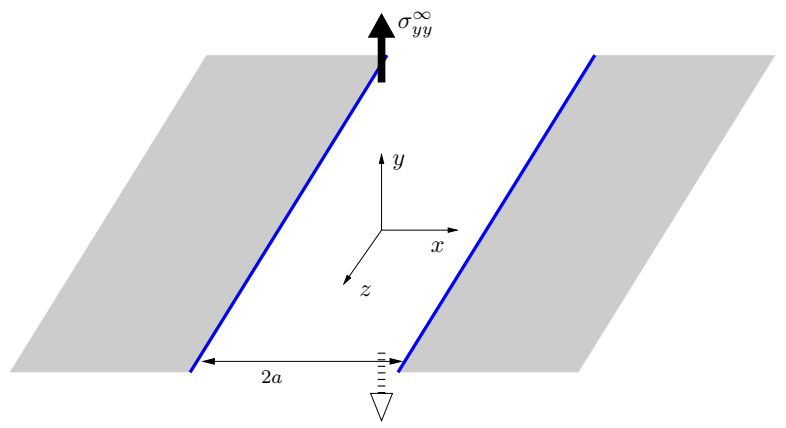

(c) An external tunnel-crack, in an infinite media loaded by remote tensile stresses

Figure 4: Several problems involving a tunnel-crack.

studied several cases involving a tunnel-crack (fig. 4) with $\partial \Omega_{u}=\varnothing$ : Leblond et al. (1996) for the tensile tunnel-crack, Lazarus and Leblond (2002c) for the shear tunnel-crack (fig. 4(a)), Pindra et al. (2010b) for two coplanar tensile tunnel-cracks (fig. 4(b)), Legrand and Leblond (2010b) for an external tunnel-crack (fig. $4(\mathrm{c}))^{5}$.

\footnotetext{
${ }^{5}$ External cracks give rise to traditional ambiguities on the external load, since they cannot withstand uniform tractions exerted at infinity. Here the situation considered unambiguously consists of two tunnel-cracks (fig. 4(b)) in the limiting case where $b \gg a$.
} 


\section{Particular model case of tensile straight crack fronts}

The aim here is to introduce formulas that are useful further on for the study of some crack propagation problems involving an initially straight crack front (for sections 4.1 and 6 in particular).

\subsection{Unperturbed geometries and loading}

For simplicity, only mode 1 is considered and $K_{1}$ is re-noted $K$. To study the propagation of a straight crack front, the most natural and simple model is the half-plane crack loaded by remote stresses (fig. 3(a)). Even if a certain number of results can be obtained with this model, it lacks crucially a lengthscale. To fill this gap, this simple model has progressively be enriched. Here, the following models are considered more specifically:

1. a half-plane crack loaded by remote stresses (fig. 3(a)), then $K(s)=K$ is a constant due to the lack of any lengthscale in this problem;

2. a half-plane crack with uniform line tractions $P$ at a distance $a$ of the front (fig. 3(b)), then $K(z)=\sqrt{\frac{2}{\pi}} P a^{-1 / 2}$;

3. a half-plane crack with uniform surface tractions $\sigma$ in a band of width $a($ fig. $3(\mathrm{c}))$, then $K(z)=2 \sqrt{\frac{2}{\pi}} \sigma a^{1 / 2}$;

4. a tunnel-crack loaded by remote stresses (fig. 4(a)) then $K(z)=$ $\sigma \sqrt{\pi} a^{1 / 2}$

All these problems can be included in the more general framework for which the initial SIF can be written under the form:

$$
K(a)=k a^{\alpha}
$$

where $k$ depends on the loading level but is independent of $a$. The value of $\alpha$ are 0 in the case $1,-1 / 2$ in case $2,1 / 2$ in cases 3 and 4 . The sign of $\alpha$ is of uppermost importance in the sequel. If $\alpha<0$ the propagation is stable under constant loading and if $\alpha>0$ unstable $^{6}$.

${ }^{6}$ This terminology makes an implicit reference to Irwin's propagation law (7) for which crack propagation occurs when the SIF reaches some critical value. For such a law and under constant loading, after the onset of crack propagation, the velocity of the crack goes immediately down to zero if the SIF decreases with distance, but continuously increases in the opposite case; hence the expressions "stable propagation ", "unstable propagation ". 


\subsection{Fourier transform of the first order variation of the SIF}

Define the Fourier Transform $\widehat{\phi}(k)$ of some arbitrary function $\phi(z)$ by

$$
\widehat{\phi}(k) \equiv \int_{-\infty}^{+\infty} \phi(z) e^{i k z} \mathrm{~d} z \quad \Leftrightarrow \quad \phi(z) \equiv \frac{1}{2 \pi} \int_{-\infty}^{+\infty} \widehat{\phi}(k) e^{-i k z} \mathrm{~d} k .
$$

Using this definition and equation (21) applied to the geometries listed in section 3.1, Fourier components $\delta \widehat{K}(k)$ of the first order variation of the mode 1 SIF $\delta K(z)$ can be written under the following form:

$$
\frac{\delta \widehat{K}(k, a)}{K(a)}=\left[\frac{\frac{\mathrm{d} K(a)}{\mathrm{d} a}}{K(a)}-a^{-1} F(p)\right] \widehat{\delta}(k)=(\alpha-F(p)) \frac{\widehat{\delta}(k)}{a}
$$

Here, $k$ is the wavenumber and $p=k a$ the dimensionless one. In the case of the tunnel-crack geometry, we have supposed that the perturbations are the same for all the fronts for simplicity, so that if we denote $\delta_{n}(z)$ the perturbation of $\mathcal{F}_{n}$, it exists a function $\delta(z)$ such as $\delta_{n}(z)=\delta(z)$ whatever $n=1, N$.

$F(p)$ can be derived from the expressions of the fundamental kernels listed in section 2.4. For instance, for the half-plane crack it reads:

$$
F(p)=\frac{p}{2}
$$

and for all the geometries of $\S 3.1$, it can be verified that (i) $F(0)=0$ and (ii) $F(p)$ increases monotonically to finally behaves as $p / 2$ for $p \rightarrow \infty$. This last behaviour is closely linked to the universal behaviour of $W_{11}\left(s, s^{\prime}\right)$ for $s^{\prime} \rightarrow s$ (eq. 15).

The general formulas for the tunnel-crack, without the symmetry hypothesis $\delta_{n}=\delta$ of the crack advance, can be found in Favier et al. (2006b). A formula similar to (33) can be find in Gao and Rice (1987b) (resp. Gao and Rice (1987a)) for an internal (resp. external) circular crack, in Pindra et al. (2010b) for two tunnel-cracks and in Legrand and Leblond (2010a) for an external tunnel-crack. For shear loading, see Gao and Rice (1986) for the half-plane crack, Pindra et al. (2008) for the interfacial half-plane crack, Pindra et al. (2010a) for the tunnel-crack. 


\section{Crack propagation in an homogeneous media}

The aim of this section is to study the crack front shape changes arising from the quasistatic propagation in an homogeneous media. First the problem of crack shape bifurcation and stability (section 4.1) is studied analytically by linear approaches, then large scale deformations (section 4.2) are presented using incremental non linear numerical simulations.

\subsection{Crack front shape linear bifurcation and stability analysis}

First order perturbation approaches are extensively used in linear bifurcation and stability analysis in various problems (Drazin, 1992; Bazant and Cedolin, 2003; Nguyen, 2000). Here the problem of configurational bifurcation and stability of a straight crack front is considered.

\subsubsection{Bifurcation}

Consider one of the model problems of section 3 and suppose that $K(z)=$ $K_{c}$ for all $z$. The configurational bifurcation problem aims at answering the following unicity question: can one find any configuration satisfying the condition that the SIF be equal to a constant along the crack front, other than the initial straight one?

The linear bifurcation problem amounts to search for a crack front perturbation $\delta(s) \neq 0$ such as the first order variation $\delta K(s)$ of the SIF is zero. By equation (33), this reads:

$$
[\alpha-F(p)] \widehat{\delta}(k)=0,
$$

so that non-zero solution exists if $[\alpha-F(p)]=0 \forall p$. Since $F(p) \geq 0$, it exists only if $\alpha \geq 0$ that is if the propagation is unstable under constant loading. The bifurcation corresponds to a sinusoidal perturbation of critical wavelength $\lambda_{c}$ solution of ( $\alpha$ has been introduced in 31):

$$
\lambda_{c}=\lambda_{c}^{*} a, \text { where } \lambda_{c}^{*}=\frac{2 \pi}{F^{-1}(\alpha)}
$$

For the half-plane crack, it corresponds to Rice (1985)'s result:

$$
\lambda_{c}=\frac{\pi K(a)}{\frac{\mathrm{d} K(a)}{\mathrm{d} a}},
$$




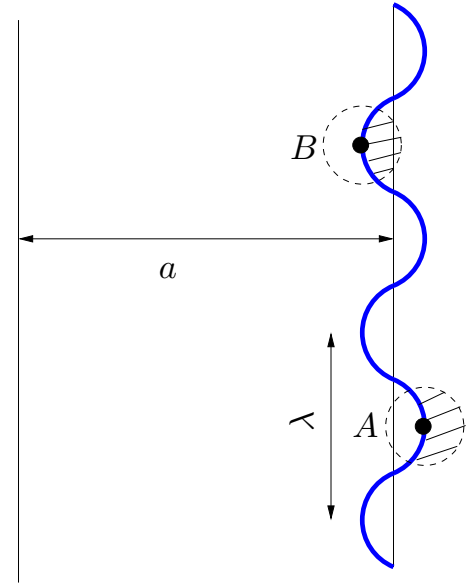

(a) Small wavelength $\lambda \ll a$ $4(\mathrm{~b}))$.

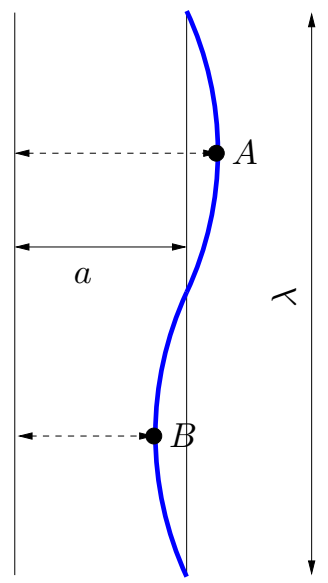

(b) Large wavelength $\lambda \gg a$

Figure 5: Sinusoidal perturbation of the crack front

which gives $\lambda_{c}=2 \pi a \sim 6.283 a$ in the case of surface tractions (fig. 3(c)). For the single tunnel-crack under remote loading, $\lambda_{c}=6.793 a$ (Leblond et al., 1996) and two interacting tunnel cracks $\lambda_{c}=18.426 a$ when $a \ll(b+a)$ (fig.

The existence of a bifurcation if $\frac{\mathrm{d} K(a)}{\mathrm{d} a}>0$ and the nonexistence if $\frac{\mathrm{d} K(a)}{\mathrm{d} a}<$ 0 has still been noticed by Nguyen (2000) in the case of thin films. It may be rationalised as follows:

1. Consider first a perturbation of the crack fronts of small wavelength, $\lambda \ll a$ (Figure 5(a)). The crack advance is maximum at point $A$ and minimum at point $B$. Draw small circles centred at these points. That part of the interior of the circle occupied by the unbroken ligament (hatched in Figure 5(a)) is larger at point $A$ than at point $B$, so that the opening of the crack is more hindered near the first point than near the second. Thus the stress intensity factors $K(A), K(B)$ at points $A$ and $B$ obey the inequality $K(A)<K(B)$.

2. Consider now a perturbation of large wavelength, $\lambda \gg a$, and again points $A$ and $B$ where the crack advance is respectively maximum and minimum (Figure 5(b)). The stress intensity factors at points $A$ and $B$ are almost the same as for ligaments of uniform width equal to 
the local width at these points (indicated by dashed double arrows in Figure 5(b)). It follows that $K(A)>K(B)$ if $\frac{\mathrm{d} K(a)}{\mathrm{d} a}>0$ and that $K(A)<K(B)$ if $\frac{\mathrm{d} K(a)}{\mathrm{d} a}<0$.

This implies:

- In the case $\frac{\mathrm{d} K(a)}{\mathrm{d} a}>0$, the difference $K(A)-K(B)$ is negative for small $\lambda$ and positive for large $\lambda$, and obviously varies continuously with this parameter. Hence some special value $\lambda_{c}$ such that $K(A)-K(B)=0$ must necessarily exist.

- In the case $\frac{\mathrm{d} K(a)}{\mathrm{d} a}<0$, the difference $K(A)-K(B)$ is negative for all $\lambda$, so that no bifurcation is possible.

In the case of shear loading, the results are more complex and can be found in Gao and Rice (1987b) for the internal circular crack, in Gao and Rice (1986) for the half-plane crack and in Lazarus and Leblond (2002b) for the tunnel-crack. For thin films, crack front bifurcation has also be studied by Nguyen (2000); Adda-Bedia and Mahadevan (2006) and observed in experiments (Ghatak and Chaudhury, 2003).

\subsubsection{Stability}

The question here is as follows: if the crack front is slightly perturbed within the crack plane, will the perturbation increase (instability) or decay (stability) in time? Equivalently, will the crack front depart more and more from its initial configuration or tend to keep it? We restrict our attention here to the cases listed in section 3 for which the $\operatorname{SIF} \mathbf{K}(z)$ in the initial configuration are uniform independent of $z$.

When the extrema of $\delta(z)$ and $\delta G(z)$ coincide, stability or instability prevails according to whether the maxima of $\delta G(z)$ correspond to the minima or maxima of $\delta(z)$ (Rice, 1985; Gao and Rice, 1986, 1987b; Gao, 1988; Leblond et al., 1996; Lazarus and Leblond, 1998b; Legrand and Leblond, 2010a). Hence the answer to the question can simply be derived from the above bifurcation discussion: sinusoidal perturbations are stable if $\alpha-F(p)<0$ that is for wavelength smaller than the bifurcation wavelength $\lambda_{c}$ (eq. 36) and unstable for $\lambda>\lambda_{c}$. In the case of non-existence of a bifurcation (stable propagation $\alpha<0$ ), stability is thus achieved whatever the wavelength. In the case $\alpha>0$, the critical wavelength is proportional to $a$, thus continuously 
increases during propagation, stability ultimately prevails for all wavelengths.

But when the extrema of $\delta(z)$ and $\delta G(z)$ do not coincide, as is for instance the case of the tunnel-crack under shear loading (Lazarus and Leblond, $2002 b$ ), it appears quite desirable to then discuss the stability issue in full generality, without enforcing such a coincidence (Lazarus and Leblond, 2002a). It is then necessary to introduce a time dependent advance law.

Let us use here the Paris law (10). Its leading term reads:

$$
\frac{\mathrm{d} a(t)}{\mathrm{d} t}=C K^{N}
$$

where $a(t)$ is the mean position of the crack front at instant $t$. Considering henceforward all perturbations as functions of the mean position $a$ of the crack instead of time $t$, one gets the first order advance equation:

$$
\frac{\partial \delta(k, a)}{\partial a}=N \frac{\delta K(k, a)}{K}
$$

which yields (Favier et al., 2006b) after use of FT (33) and integration $\left(a_{0}\right.$ denotes the initial value of $a$ ):

$$
\frac{\widehat{\delta}(k, a)}{\widehat{\delta}\left(k, a_{0}\right)}=\exp \left[N \int_{k a_{0}}^{k a}(\alpha-F(p)) \frac{\mathrm{d} p}{p}\right]
$$

or using the property $F(0)=0$ :

$$
\frac{\widehat{\delta}(k, a)}{\widehat{\delta}\left(k, a_{0}\right)}=\left(\frac{a}{a_{0}}\right)^{N \alpha}\left(\frac{\psi(k a)}{\psi\left(k a_{0}\right)}\right)^{N \alpha}
$$

where $\psi(p)$ is defined by:

$$
\psi(p)=\exp \left[-\int_{0}^{p} \frac{F(q)}{q} \mathrm{~d} q\right]
$$

For the half-plane crack, its value is $\psi(p)=\exp \left(-\frac{p}{2}\right)$, for the tunnel-crack it can be found in Favier et al. (2006b). For the sequel, it is useful to note that whatever the geometry (half-plane or tunnel), this function $\psi(p)$ decreases from 1 to 0 when $p$ varies from 0 to $+\infty$.

From equation (40), it is clear that: 
- If $\frac{\mathrm{d} K(a)}{\mathrm{d} a}<0$, then $\alpha-F(p)<0$ so that all Fourier components of any wavelength decrease with crack growth $a$.

- If $\frac{\mathrm{d} K(a)}{\mathrm{d} a}>0$, for any given $k,|\widehat{\delta}(k, a)|^{2}$ increases as long as $a$ remains smaller than $\frac{2 \pi}{k \lambda_{c}^{*}}$ and decreases afterwards.

For $t \rightarrow \infty$, one can show that:

$$
\left\{\begin{array}{l}
\text { For } k=0, \quad\left|\frac{\widehat{\delta}(0, a)}{\widehat{\delta}\left(k, a_{0}\right)}\right|^{2}=\left(\frac{a}{a_{0}}\right)^{2 N \alpha} \\
\text { For } k \neq 0, \quad\left|\frac{\widehat{\delta}(k, a)}{\widehat{\delta}\left(k, a_{0}\right)}\right|^{2} \sim\left(\frac{a}{a_{0}}\right)^{2 N \alpha} \exp (-N|k| a) \rightarrow 0
\end{array}\right.
$$

so that:

- If $\frac{\mathrm{d} K(a)}{\mathrm{d} a}<0$, any initial perturbation disappears.

- If $\frac{\mathrm{d} K(a)}{\mathrm{d} a}>0$, the moduli of all Fourier components ultimately decay, except for $k=0$ which continuously increases. This phenomenon is due to the fact that for all values of $\lambda$ except $+\infty, \lambda$ always ultimately becomes smaller than $\lambda_{c}(a)$ since the former wavelength is fixed whereas the latter increases in proportion with $a$.

Thus, one can conclude that whatever the small perturbation of crack front, the initial configuration is finally retrieved ${ }^{7}$. In the case of stable crack propagation $\frac{\mathrm{d} K(a)}{\mathrm{d} a}<0$, the stability prevails at all lengthscales from the beginning. In the case of unstable crack propagation $\frac{\mathrm{d} K(a)}{\mathrm{d} a}>0$, instability first prevails for all lengthscales such as $\lambda>\lambda_{c}$, but since $\lambda_{c}$ is a growing function of the crack advance $a$, all wavelengths finally becomes stable so that the perturbation finally disappears. This is true for all the problems listed in section 3 provided that the first order study stays valid. To extend them to large perturbations, higher order terms must be taken into account. This is the subject of next section.

\footnotetext{
${ }^{7}$ the wavelength $k=0$ corresponds indeed to a infinite wavelength that is an almost straight crack front.
} 


\subsection{Largescale propagation simulations}

In the previous sections, the perturbation approach was applied to small perturbations of the crack front. Following an original idea of Rice (1989), Bower and Ortiz (1990) first extended the method to the study of arbitrary large propagation of a tensile crack leading the way to the numerical resolution of some complex three dimensional crack problems. It consists in applying numerically the perturbation approach described in section 2 , to a succession of small perturbations arising in arbitrary large ones. The media is assumed to be infinite loaded by remote stresses so that the SIF can be updated using formula (20) and the FKs using formula (24). The crack front shape at each instant is obtained by the inversion of heavily implicit systems of equations resulting from the direct application of Irwin's criterion (7). The method was then extended and simplified by Lazarus (2003); notably a unified Paris' type law (10) formulation for fatigue and brittle fracture $(N \rightarrow+\infty)$ is proposed that gives the advance of the crack front in explicit form once the SIF is known. Extension to shear loading is performed in Favier et al. (2006a).

Concerning propagation in an homogeneous media, Lazarus (2003) studied the asymptotic behaviour of the SIF near an angular point of the front and retrieved the theoretical results of Leblond and Leguillon (1999) about the SIF singularity around a corner point of the front, the fatigue and brittle propagation paths of some special crack shapes (elliptical, rectangular, heart shaped ones) (fig. 6) loaded by remote tensile stresses. It appears that in all the cases studied, the crack becomes and stays circular after a certain time emphasizing that among all the configurations studied only the circular crack shape is stationnary. In the case of shear loading (Favier et al., 2006a), it appears (fig. 7) that the stationnary shape is nearly elliptic, the ratio of the axes being well approximated by:

$$
\frac{a}{b}=(1-\nu)^{\frac{\beta}{\beta+1}}
$$

$\beta$ is the Paris law exponent in mixed mode loading (9), $b$ corresponds to the axis in the direction of the shear loading. Whether all embedded plane cracks tend toward a configuration with uniform value of $\mathcal{G}(s)$ is a general result, has however, to my best knowledge, not been demonstrated, even if one guess that energy minimisation is the physical ground. 


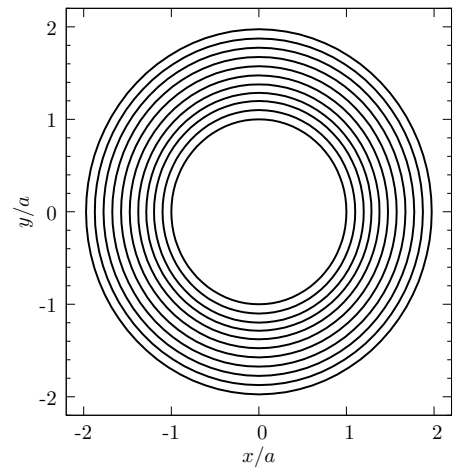

(a)

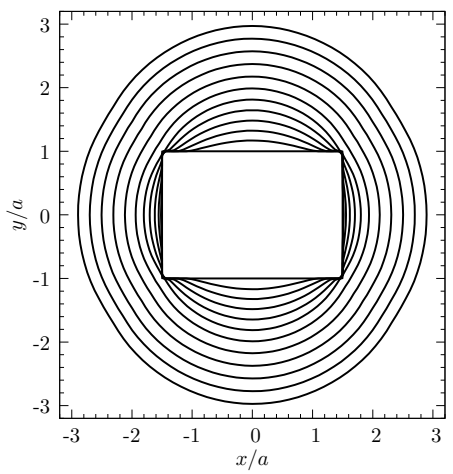

(c)

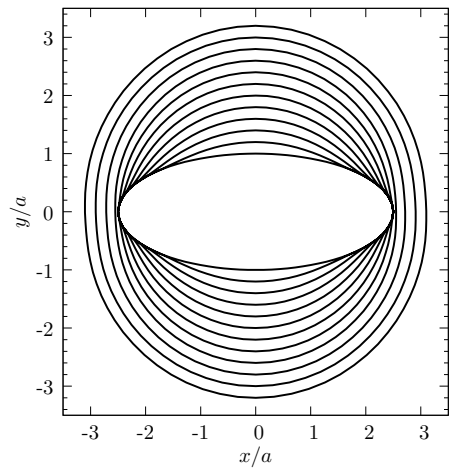

(b)

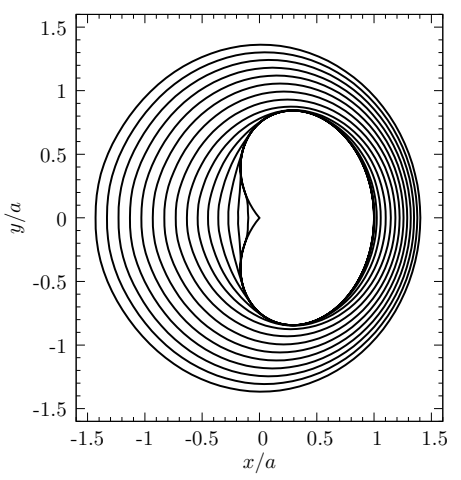

(d)

Figure 6: Successive crack fronts of pure tensile mode cracks in brittle fracture (eq. 10 with $N=50$ ). Similar figures for fatigue can be found in Lazarus (2003) and show the same circular stationnary crack shape.

\section{Crack trapping by tougher obstacles}

In previous section, all the material constants (elasticity coefficients, fracture toughness) were supposed to be homogeneous throughout the media. In the sequel, we are still considering the problem of a crack propagating in the slow velocity regime as in section 4 but with the toughness becoming heterogeneous. The elasticity coefficients are supposed to remain constant so that the perturbation approach of section 2 remains valid. If the toughness is heterogeneous, the crack advance changes from point to point and the crack front shape changes during propagation even if the SIFs were initially uniform. In this section, the propagation of the front through well defined 


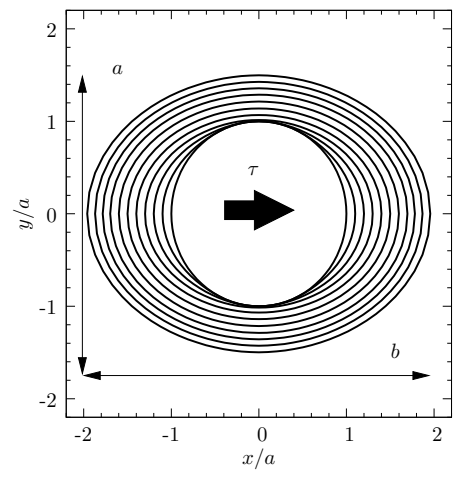

(a)

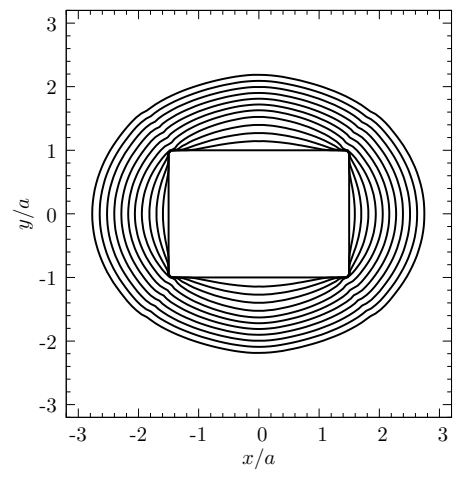

(c)

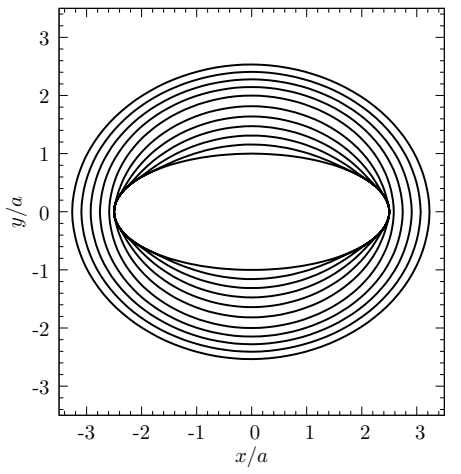

(b)

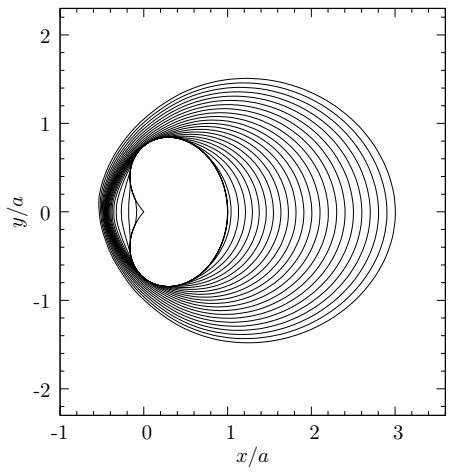

(d)

Figure 7: Successive crack fronts of pure shear mode cracks in brittle fracture (eq. 9 with $\left.\beta=25, \mathcal{G}_{0}=0\right)$. Shear is along the $x$-axis. Similar figures for fatigue can be found in Favier et al. (2006a) and show also a quasielliptic stationnary crack shape.

tougher obstacles is studied. In section 6, the toughness is supposed to be disordered so that a statistical approach is necessary.

Tougher inclusions may prevent or hinder the final breaking of a solid by two mechanisms: crack bridging and crack front trapping. The mechanism of toughening referred to as bridging occurs when unbroken inclusions lag behind a main crack front hindering its opening by pinning or friction; and as trapping when the crack front is deformed when it penetrates into the tougher zone or bows out it (Lange, 1970).

Whereas some aspects of crack bridging can be studied by 2D elasticity problems (Budiansky et al., 1988), crack trapping induces crack front shape deformations that makes the elasticity problem fully 3D. To understand the 
mechanism, let us consider a tunnel-crack loaded by remote tensile loading $\sigma$ (fig. 4(a)) . The SIF for the straight-crack front then reads:

$$
K=\sigma \sqrt{\pi a}
$$

In the absence of obstacles, the propagation is unstable under constant loading so that the breakdown of the solid occurs as soon as the threshold is reached, unless the loading is decreased to ensure that $K(s) \leq K_{c}$ at each instant: $\sigma=K_{c} / \sqrt{\pi a}$. In the presence of tougher $\left(K_{c}^{p}>K_{c}^{m}, K_{c}^{p}, K_{c}^{m}\right.$ being resp. the matrix and particles toughness), the crack propagation of a tunnel-crack of width $2 a_{0}$ starts when $\sigma=\sigma_{0}$, where $\sigma_{0}=K_{c}^{m} / \sqrt{\pi a_{0}}$. Then,

- either the spacing between the obstacles is large enough, so the SIF in the matrix still increases at constant loading. The final breakdown then occurs for $\sigma=\sigma_{0}$.

- either the spacing is small enough, so the SIF in the matrix decreases at constant loading. Then a transient period of stable propagation at constant loading exists, so that the unstable propagation loading, that is the breakdown one, is increased.

In this last case, the breakdown may appear in two different situations:

1. The crack front has penetrated under a stable manner into the obstacles until reaching a configuration such as $K(s)=K_{c}(s)$ for all $s$ at unstable breakdown instant. The corresponding loading level $\sigma_{c}$ can then be determined by the following relation derived by Rice (1988); Gao and Rice (1989):

$$
\frac{\sigma_{c}^{2}}{\sigma_{0}^{2}}=\frac{<K_{c}^{2}>}{\left(K_{c}^{m}\right)^{2}}
$$

This situation is called "regular" by Gao and Rice (1989) and "weak pinning" by Roux et al. (2003). It appears for not too large toughness differences (to ensure not too large crack front deformations) and particles that are large enough in the direction of propagation (to have the time to reach the equilibrium position). First order simulations of this regime have been performed by Gao and Rice (1989) using the perturbation approach described in section 2.2. The results have been compared to simulations of the same problem performed using a Boundary Element Method showing the accuracy and the limits of the first 
order approach. They have also been compared to experiments by Dalmas et al. (2009): good agreement between the theoretical and experimental crack shapes have been shown. The work of Gao and Rice (1989) realized in mode 1 is extended to modes 2 and 3 in Gao et al. (1991). Numerical large scale propagation simulations of this case have also been performed by Bower and Ortiz (1990, 1991, 1993) for the half-plane crack and Lazarus (2003) for the circular one.

2. The unstable breakdown occurs before $K(s)=K_{c}(s)$ is reached for all $s$, so that only a part of the front propagates at the breakdown instant, $K(s)$ being lower than $K_{c}(s)$ on the other part. In this situation, called "irregular" by Gao and Rice (1989), "unstable" by Bower and Ortiz (1991), and "strong pinning" by Roux et al. (2003) the value of $\sigma_{c}$ can only be determined numerically in each particular case. It has been done by Bower and Ortiz (1991), using the incremental method described in section 4.2 , for a half plane crack propagating through an array of particles. Their results concerning the bow out of the crack front segments beyond an unbroken particle when the toughness of the particles is high enough to prevent the penetration of the front in the particle are compared to experiments by Mower and Argon (1995) and show good agreement in general.

\section{Crack propagation in a disordered media}

In the last two decades, a number of works have been devoted to the study of the evolution in time of the shape of the front of planar cracks propagating in mode 1 in an elastic solid with randomly variable fracture toughness. These works can be roughly divided into three groups. The first group includes the works of Perrin and Rice (1994), Ramanathan and Fisher (1997) and Morrissey and Rice (1998, 2000). They were devoted to the theoretical study of some statistical features of the geometry of the front of a tensile half-plane crack propagating dynamically. The second group of papers consists of some experimental studies of the evolution in time of the deformation of the front propagating quasistatically; see e.g. Schmittbuhl and Màløy (1997), Delaplace et al. (1999), Schmittbuhl and Vilotte (1999), Schmittbuhl et al. (2003a). The third group studied statistical properties of the shape of crack fronts for a straight crack (half-plane or tunnel-crack) propagating quasistatically: on the one hand, Schmittbuhl et al. (1995) and Katzav and Adda-Bedia (2006) focusing notably on self-affine properties of 
Now introduce some small quenched (independent of time at a give material point) fluctuations of the toughness :

$$
K_{c}(z, x)=\bar{K}_{c}(1+\kappa(z, x)), \quad|\kappa| \ll 1
$$


It produces small fluctuations $\delta(z, a(t))$ and $\delta K(z, a(t))$ of the crack front position $a(z, t)$ and of the SIF $K(z, t)$ around its mean values $a(t)$ and $K(a(t))$ so that :

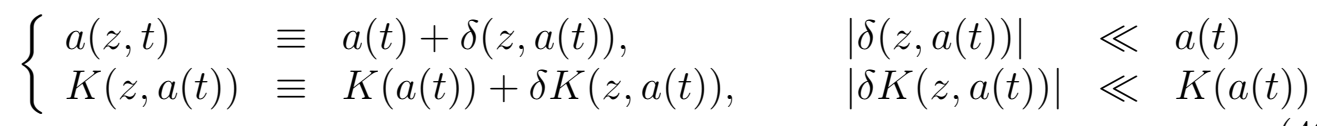

Expanding Irwin's criterion (7) to first order, identifying terms of order 0 and 1 and replacing the kinematical time $t$ by the mean crack position $a$, one gets :

$$
\left\{\begin{array}{l}
K(a)=\bar{K}_{c} \\
\frac{\delta K(z, a)}{K(a)}=\kappa(z, a)
\end{array}\right.
$$

It shall be noticed that in this first order expansion, the quenched fluctuations are transfered on the mean crack position so that this approach does not permit to distinguish between annealed (time dependent fluctuations at a given position) and quenched noise.

Now, taking the Fourier transform of the equation (50.2) and using equation (33) giving the first order variation of the SIF as a function of the crack perturbation, one gets:

$$
\hat{\delta}(k, a)=\frac{a \hat{\kappa}(k, a)}{\alpha-F(k a)}
$$

Unfortunately, if $\alpha>0$, the expression (51) is meaningless because the FT is divergent for $p$ such as $\alpha-F(p)=0$. This is linked to the existence of bifurcations (see section 4.1). We shall therefore consider the sole case of stable $2 \mathrm{D}$ crack propagation $(\alpha<0)$ henceforward. Eq. (51) then takes the form:

$$
\hat{\delta}(k, a)=-\frac{a \hat{\kappa}(k, a)}{|\alpha|+F(p)}
$$

This equation allows to obtain the first order crack front fluctuations $\delta$ for any given small toughness fluctuation $\kappa$. Notice that it is entirely determined by the instantaneous distribution of the toughness. It may be used to study the shape deformations during trapping by tougher obstacles. In the sequel, however we suppose the material to be disordered so that only statistical properties of $\kappa$ are known and statistical study becomes necessary. 
6.1.2. Power spectrum of the crack front fluctuations versus toughness fluctuations

From equation (52), one gets for the power spectrum $\widehat{\mathcal{A}}(k, a)$ of the fluctuation $\delta(z)$ of the crack front:

$$
\widehat{\mathcal{A}}(k, a)=a^{2} \frac{\widehat{\mathcal{K}}(k)}{(|\alpha|+F(p))^{2}}
$$

where $\widehat{\mathcal{K}}(k)$ is the power spectrum associated with the toughness fluctuations $\kappa$ supposed statistical homogeneous so that $\widehat{\mathcal{K}}(k)$ is independent of $a$. This expression is quiet general. Some properties of it, in the particular case of uncorrelated fluctuations are given in the sequel.

In the case of white noise $\widehat{\mathcal{K}}(k, a)=\widehat{\mathcal{K}}_{0}$, equation (53) gives $\frac{\widehat{\mathcal{A}}(k, a)}{\widehat{\mathcal{K}}_{0} a^{2}}$ under an analytical form:

$$
\frac{\widehat{\mathcal{A}}(k, a)}{\widehat{\mathcal{K}}_{0} a^{2}}=\frac{1}{(|\alpha|+F(p))^{2}}
$$

It is plotted as a function of $p=k a$ in figure 8(a) for several values of $\alpha$ and a tunnel or a half-plane crack. In all these cases, one can notice the presence of two regimes, with a transition between them depending on the crack geometry and on the loading: one universal regime (independent of the geometry) for $p=k a \gg 1$ that is small wavelengths $\lambda \ll a$ where $\frac{\widehat{\mathcal{A}}(k, a)}{\widehat{\mathcal{K}}_{0} a^{2}}$ decreases with $k a$ and a second (geometry dependent one) for $p=k a \ll 1$ that is large wavelengths $\lambda \gg a$ corresponding to a saturation. The existence of this second regime is closely linked to the finite size of the system and can not be obtained by the model of a half-plane crack loaded by remote tensile stresses that, we recall, lacks any lengthscale.

One can notice that such a behaviour (see Barabási and Stanley (1995)) corresponds to a Family and Vicsek (1985) scaling defined by $\widehat{\mathcal{A}}(k, a)=$ $a^{\frac{1+2 \zeta}{\tau}} G\left(k a^{1 / \tau}\right)$ where $G(x)$ is constant for $x \ll 1$ and $G(x) \sim x^{-1-2 \zeta}$ for $x \gg 1$. Comparison with (54) gives indeed:

$$
\begin{aligned}
& G(x)=\widehat{\mathcal{K}}_{0}(|\alpha|+F(x))^{-2} \\
& \zeta=0.5 \text { (roughness exponent) and } \tau=1 \text { (dynamic exponent). }
\end{aligned}
$$

To better understand the Family and Vicsek (1985) scaling, let us study the dependence over $k$ and $a$ of $\frac{\widehat{\mathcal{A}}(k, a)}{\widehat{\mathcal{K}}_{0}}$ and consider the particular case of a 


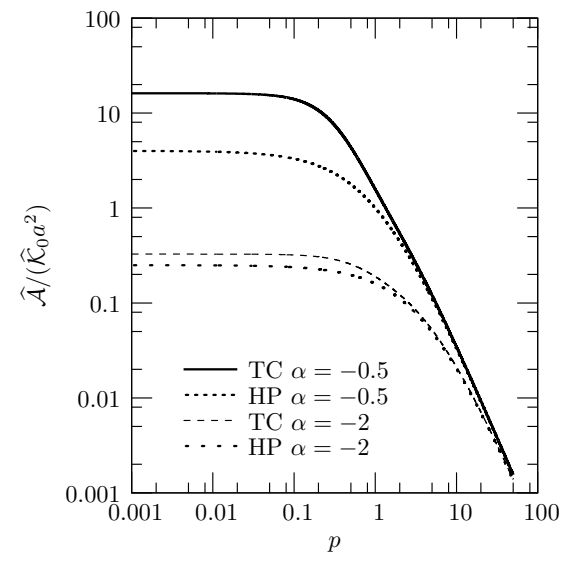

(a) Master curves for different geometries $\quad(\mathrm{TC}=$ tunnel-crack, $\mathrm{HP}=$ halfplane crack) and values of $\alpha$.

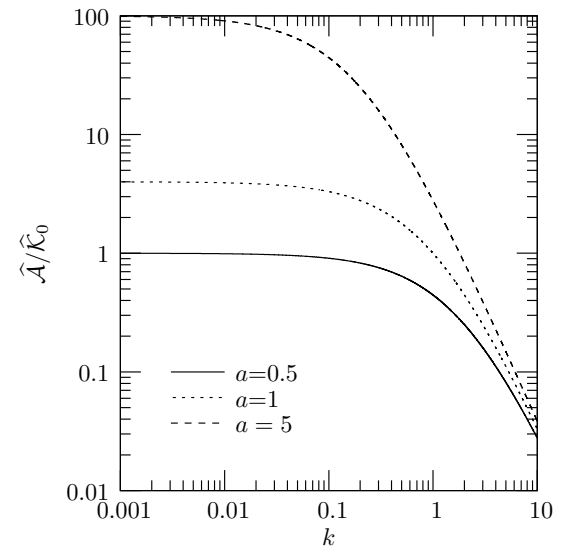

(b) Dependence toward $k$ for several given values of $a$ (half-plane crack with $\alpha=-1 / 2)$.

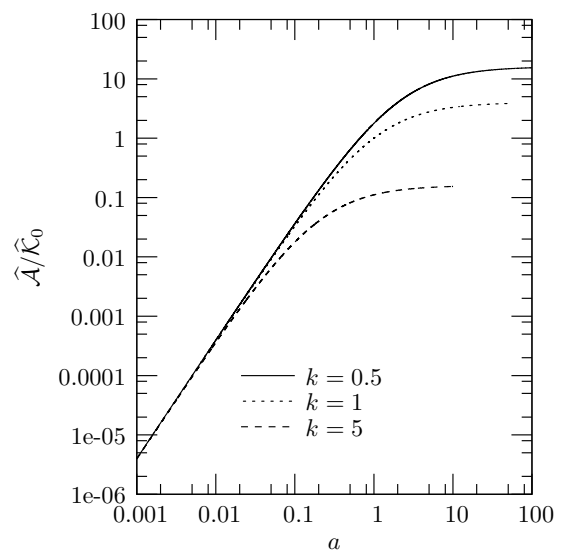

(c) Dependence toward $a$ for several given values of $k$ (half-plane crack with $\alpha=-1 / 2$ ).

Figure 8: Power spectrum of the crack front fluctuations for white noise toughness fluctuations.

half-plane crack with $\alpha=-1 / 2$. One can notice on figure $8(\mathrm{~b})$, that for a given value of the mean position $a$ :

- The large wavelength components are more developed than the small ones and are constant above a certain threshold. 
- When $a$ increases, the large wavelength components increases, but the small ones are steadystate and more and more components are in this last steadystate regime.

One can notice on figure 8(c), that for a given value of $k$, that is $\lambda$ :

- The components increases with $a$ until reaching a saturation.

- The increasing regime rate is similar whatever the wavelength, but when $k$ decreases, that is $\lambda$ increases, the increasing regime lasts longer so that the final amplitude increases with wavelength.

One can also derive the asymptotic expressions of the power spectrum $\widehat{\mathcal{A}}(k, a)$ :

$$
\widehat{\mathcal{A}}(k)=\frac{4 \widehat{\mathcal{K}}(k)}{k^{2}} \text { for } k \neq 0 \quad \text { and } \widehat{\mathcal{A}}(0)=\frac{\widehat{\mathcal{K}}(0) a^{2}}{\alpha^{2}} \text { for } k=0
$$

One shall notice that the convergence is not uniform so that the asymptotic behaviour of the autocorrelation function can not be obtained by simply inversion of the asymptotic behaviour of its Fourier transform. For the results concerning the autocorrelation or related function as the square fluctuations, the reader is invited to reefer at Pindra et al. (2008) or to the table 1. One shall however notice that relation (53) is the cornerstone for such a derivation. It shall also allow to perform numerical simulations of the evolution of the power spectrum or the functions related to the autocorrelation by inverse Fourier Transform if the toughness fluctuation power spectrum is given. Such developments is left for further work.

Similar results for an interfacial half-plane crack have been derived by Pindra et al. (2008): the mismatch of elastic properties between the materials introduces oscillations in the longtime behaviour but no significant difference in the mean behaviour. The case of a shear tunnel-crack has been considered by Pindra et al. (2010a): the results are rather similar to those previously obtained for mode 1; one novelty, however, is that, the fronts no longer tend to become symmetrical in time as in mode 1 (Favier et al., 2006b), so that correlations between crack front fluctuations at two points are higher for points located on the same front than for points located on distinct ones. 


\subsection{Subcritical or fatigue propagation}

Let us suppose now that the crack advance is given by Paris' law (10). The inhomogeneity of the material is modelled by assuming the Paris constant to slightly fluctuated around its mean value; the Paris exponent $N$ being considered as uniform for simplicity:

$$
C(z, x)=C(1+\delta c(z, x)), \quad|\delta c(z, x) \ll 1|
$$

It produces small fluctuations $\delta(z, a(t))$ and $\delta K(z, a(t))$ of the crack position and the SIF around its mean position $a$ (eq. 49).

\subsubsection{Evolution of the perturbation of the crack front}

Expanding first the propagation law to first order in $\delta(z, t), \delta K(z, t)$ and identifying terms of order 0 and 1 , one gets

$$
\left\{\begin{array}{l}
\frac{d a}{d t}(t)=C[K(t)]^{N} \\
\frac{\partial \delta}{\partial t}(z, t)=C[K(t)]^{N}\left[N \frac{\delta K(z, a(t))}{K(t)}+\delta c(z, a(t))\right] .
\end{array}\right.
$$

Eliminating $d t$ between these expressions and considering henceforward all perturbations as functions of the mean position $a(t) \equiv a$ of the crack instead of time, one gets

$$
\frac{\partial \delta}{\partial a}(z, a)=N \frac{\delta K(z, a)}{K(a)}+\delta c(z, a) .
$$

Upon use of the Fourier decompositions of $\delta(z, a), \delta K(z, a), \delta c(z, a)$ and equation (33), this finally yields the evolution equation of the Fourier transform $\widehat{\delta}(k, a)$ of the perturbation of the crack front:

$$
\frac{\partial \widehat{\delta}}{\partial a}(k, a)=\frac{N}{a}[\alpha-F(k a)] \widehat{\delta}(k, a)+\widehat{\delta c}(k, a) .
$$

Assuming the crack to be initially straight and integrating the linear, inhomogeneous, first-order differential equation (58) by standard methods, one gets

$$
\widehat{\delta}(k, a)=\int_{a_{0}}^{a}\left(\frac{a}{a^{\prime}}\right)^{N \alpha}\left[\frac{\psi(k a)}{\psi\left(k a^{\prime}\right)}\right]^{N} \widehat{\delta} c\left(k, a^{\prime}\right) \mathrm{d} a^{\prime}
$$

where $a_{0}$ denotes the initial value of $a$ and $\psi$ the function defined by equation (42). 
Notice that contrary to the brittle case (eq. 52), this equation is valid whatever the sign of $\alpha$ and that an effect of memory of previous configurations of the crack front is present here. This equation allows to obtain the crack front fluctuations $\delta$ for any given Paris' constant fluctuation $\delta c$. This shall be done numerically and is leaved for a further work. In the sequel, however only statistical properties of $\delta c$ are known, so that statistical study becomes necessary.

\subsubsection{General formula for the power spectrum of the perturbation of the crack front}

By the expression (59) of $\widehat{\delta}(k, a)$, one gets for the power spectrum of the crack front fluctuations:

$$
\widehat{\mathcal{A}}(k, a)=\int_{a_{0}}^{a} \int_{a_{0}}^{a}\left(\frac{a^{2}}{a_{1} a_{2}}\right)^{N \alpha}\left(\frac{[\psi(k a)]^{2}}{\psi\left(k a_{1}\right) \psi\left(k a_{2}\right)}\right)^{N} \widehat{\mathcal{C}}\left(k, a_{2}-a_{1}\right) \mathrm{d} a_{1} \mathrm{~d} a_{2},
$$

where $\widehat{\mathcal{C}}\left(k, a_{2}-a_{1}\right)$ is the power spectrum of the Paris' constant fluctuations $\delta c$.

Due to the memory effect, this equation is more complex than the equivalent (53) one in brittle fracture. Its properties for any value of $a$ has not at present been studied. Its asymptotic behaviour for $a \rightarrow \infty$ has however be obtained by Favier et al. (2006b) and Pindra et al. (2008). For $k=0$, one gets:

$$
\widehat{\mathcal{A}}(0, a) \sim\left\{\begin{array}{l}
\frac{\widetilde{\mathcal{C}}(0,0)}{2 N \alpha-1} a_{0}\left(\frac{a}{a_{0}}\right)^{2 N \alpha} \text { if } \alpha>\frac{1}{2 N}, \\
\frac{\widetilde{\mathcal{C}}(0,0)}{1-2 N \alpha} a \text { if } \alpha<\frac{1}{2 N},
\end{array}\right.
$$

and for $k \neq 0$ :

$$
\widehat{\mathcal{A}}(k, a) \sim \frac{\widetilde{\mathcal{C}}(k, 0)}{N|k|}
$$

where $\widetilde{\mathcal{C}}\left(k, k^{\prime}\right)$ is the double $z, x$-Fourier transform of the function $\mathcal{C}$.

One can notice, like in brittle fracture that the large wavelengths are preferentially selected by the system and that ultimately only the zero wavenumber $k=0$ component still evolves with $a$, the other components being in a steadystate (independent of $a$ ) rough regime. The first order study is not sufficient to determine the roughness exponent in this case (it gives indeed $\zeta=0)$. This is probably linked to the memory effect that delay the development of this regime. A second order study is then necessary. It has 
579 been performed by Katzav and Adda-Bedia (2006) who obtains a roughness ${ }_{580}$ exponent of $\zeta=0.5$.

581 6.3. Synthesis of the theoretical analytical results

${ }_{582}$ The previous results derived for the half-plane or tunnel crack, with ad-

583 ditional ones derived from Favier et al. (2006b) and Pindra et al. (2008) are 584 summarized in table 1. 


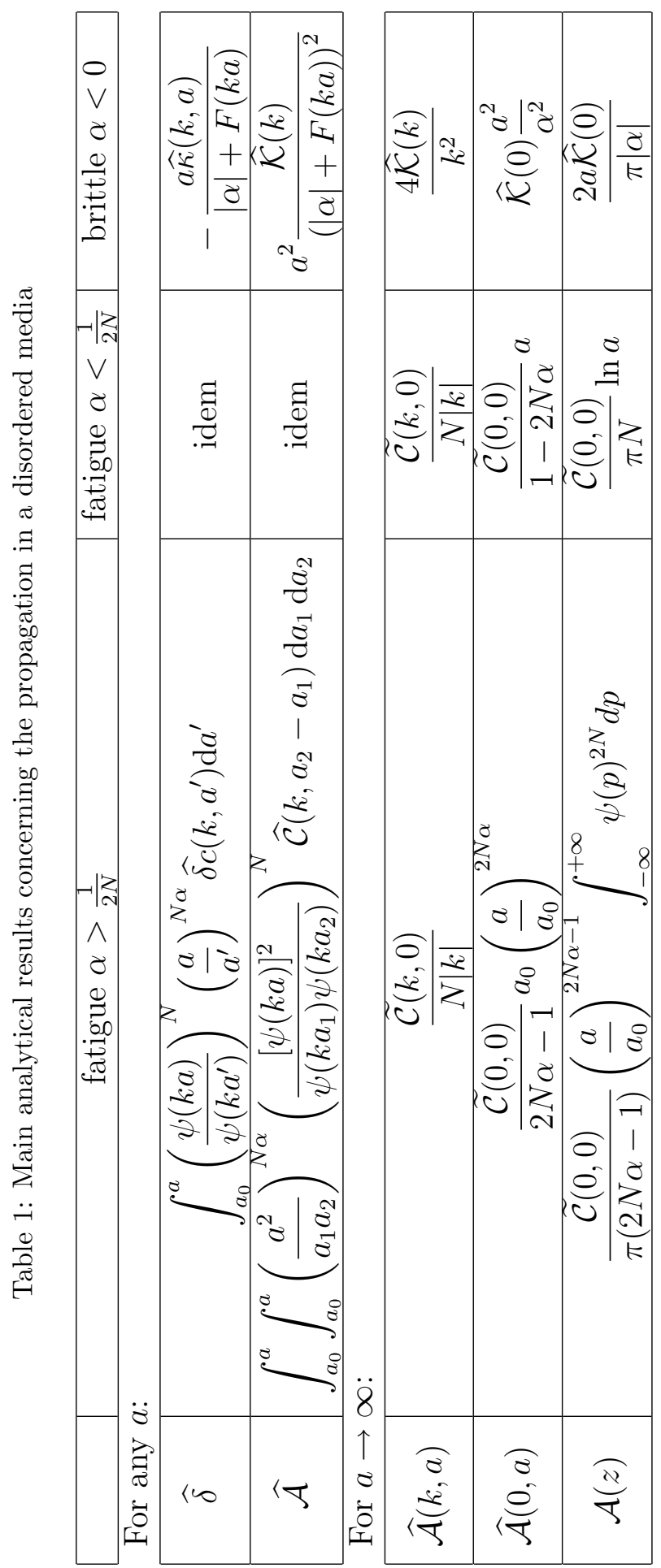


We recall that in this table $F(p)$ is the function introduces in $\S 3.2$. This function is such as $F(0)=0$ and increases monotonically to finally behaves as $p / 2$ for $p \rightarrow \infty$. The function $\psi$ is defined by eq. (42) and decreases from 1 to 0 when $p$ varies from 0 to $+\infty$.

One notices that in all the cases the system preferentially "selects" perturbations of the crack front with small wavenumbers $k$, that is, large wavelengths $\lambda=2 \pi /|k|$. Physically it is link to the process explained in $\S 4.1$. One can also easily discuss, using the table 1 , the differences between brittle fracture and fatigue, the role of the loading type ( $\operatorname{sign}$ of $\alpha$ ) and of the crack geometry (function $F(p)$ and $\psi$ ):

- Concerning the crack advance law, one can notice by comparison of columns 3 and 4, that the relations are less complex in brittle fracture than in fatigue since their is no time dependence of the response in the first case contrary to the second. One can also notice that the disorder grows faster in brittle fracture than in fatigue, the development being slowed down by a memory effect. And finally, for $\alpha>0$, the treatment is possible only in fatigue, since in brittle fracture the appearance of bifurcation renders the problem ill-posed.

- Thus, the dependence upon the sign of $\alpha$ can be considered only in fatigue. Comparison of columns 2 and 3 shows that the disorder grows faster for $\alpha>\frac{1}{2 N}$ than for $\alpha<\frac{1}{2 N}$. It is obvious since instable wavelengths exists for $\alpha>0$ and not for $\alpha<0$. The selection of the large wavelengths remains however since the large ones grow faster than the small ones.

- Concerning the crack geometry, one can notice that the asymptotic behaviour for $a \rightarrow \infty$ is independent of $F$, that is the same for the half-plane and tunnel cracks. Moreover, we have seen in section 6.1.2, that in brittle fracture, the power spectrum satisfies a Family-Vicsek scaling in both cases and the geometry introduces a difference only for the transition toward the large wavelength saturation regime (fig. $8(\mathrm{a})$ ).

\subsection{Brittle fracture: case $\exists x, z$ such as $K(x, z)<K_{c}(x, z)$ (strong pinning)}

In the case of strong pinning, when points of the crack front such as $K(x, z)<K_{c}(x, z)$ exist, an analytical approach is no more possible. Using a numerical approach based on the perturbation approach for the half-plane 
crack of Rice (1985), Schmittbuhl et al. (1995); Schmittbuhl and Vilotte (1999); Rosso and Krauth (2002) have obtained a different value of the roughness exponent: $\zeta \sim 0.35-0.4$. In these simulations, the toughness fluctuations were supposed to be quenched.

The reason for the difference between the analytical value $\zeta \sim 0.5$ obtained for weak pinning and this last result shall be clarified. It shall be due to the difference weak/strong pinning, to the difference quenched/annealed still noticed in other problems (Kardar, 1998) but also to numerical biases. The analytical results may for this last point serve as validation for the numerical procedures.

\subsection{Synthesis of the major results}

Now, let us list the factors that have an influence on the crack fluctuations resulting from toughness ones. Among them, we have seen that some of them have a quantitative influence on the statistical properties of the crack fluctuations, but a minor influence on the qualitative type of behaviour:

- Whether the crack propagates in an homogeneous media or along an interface (Pindra et al., 2008);

- Whether the loading is in tensile or shear mode (Pindra et al., 2010a);

- Whether the FK is the one of a half-plane or a tunnel-crack.

Some others have a major influence, namely:

- The advance law: Paris' law gives nonlocal time-dependent laws for the evolution of the crack fluctuation in contrary to Irwin's criterion. Moreover, strong pinning (see $\S 5$ for the definition) seems to lead to different value of the roughness exponent: $\zeta \sim 0.35-0.4$ (Schmittbuhl et al., 1995; Rosso and Krauth, 2002) then weak pinning $\zeta \sim 0.5$.

- The loading: we have seen previously the dependance of the result with the sign of $\frac{\mathrm{d} K(a)}{\mathrm{d} a}$.

- Uncorrelated/correlated fluctuations: equation (53) shows the dependance of the result with the toughness fluctuations (see also Schmittbuhl and Vilotte (1999)).

- Annealed/quenched noise: has been shown to give different results in other problems (Kardar, 1998). 
The model is however valid only:

- In the quasistatic case. When dynamic effects become important, one may refer for instance to Ramanathan and Fisher (1997) or Morrissey and Rice (1998).

- Outside the Process Zone. Inside percolation damage models have been used (Hansen and Schmittbuhl, 2003).

- For small fluctuation variations. No full description of large variations has until now been developed.

- Far from any boundary. Interactions with boundaries have been show to be important, but have seldom be studied (Gao et al. (1991), Pindra et al. (2010b), Legrand and Leblond (2010b)).

Due to the difficulty of observation of the crack front during the propagation, few different experiments exist at present, that allow to study the crack front deformations. Among them Daguier et al. (1995) used ink injections to follow the crack front in brittle fracture and fatigue. Schmittbuhl and Màløy (1997) studied the in-plane propagation through a transparent plexiglas block, the toughness fluctuations being obtained by sand blasting the surface of two plexiglas plates before to weld them together. Despite the numerous papers written on the subject (Schmittbuhl and Màløy, 1997; Delaplace et al., 1999; Maloy and Schmittbuhl, 2001; Schmittbuhl et al., 2003b; Hansen and Schmittbuhl, 2003; Schmittbuhl et al., 2003a; Santucci et al., 2009), the debate seems not closed. All the previous points shall be verified to clarify the situation.

\section{Conclusion}

Crack front small perturbation approach initiated by Rice (1985) and later extended to more complex cases has been recalled. This approach allows to update the stress intensity factors when the crack front is slightly perturbed in its plane. Applications concerning the deformation of the crack front when it propagates quasistatically in an homogeneous or heterogeneous media have been considered in brittle fracture or fatigue/subcritical propagation. Only the case of one crack propagating in an infinite plane without interaction with a boundary has been considered. The stable shapes corresponding to uniform SIF have been derived: straight or circular, but also 
when bifurcations exists, wavy crack fronts. For a straight crack, it has been shown that perturbation of all lengthscales progressively disappears unless disordered fracture properties yields Family and Vicsek (1985) roughness of the crack front.

This approach has recently be extended to interaction between several cracks: the FK for two tunnel-cracks has been derived (Pindra et al. (2010b), Legrand and Leblond (2010b)) and the disorder during their coalescence studied (Legrand and Leblond, 2010a). Interestingly, it has been shown that, stability first prevails for all lengthscales such as $\lambda<\lambda_{c}$, but since $\lambda_{c}$ is this time a decreasing function of the crack advance, all wavelengths finally become unstable so that the perturbation does not vanish. This underlines that stability results depend on the interaction of the crack with other cracks and more generally also, with obstacles or solid boundaries. Such a conclusion has still been obtained by Gao et al. (1991) who studied the stability issue when the front approaches a stress free plate boundary: when the crack is far enough from the boundary, the critical wavelength $\lambda_{c}$ increases with crack growth (the model of infinite solid is then valid) and when it approaches the boundary, $\lambda_{c}$ decreases with crack growth.

Now, comparison of the theoretical results recalled in this paper with experiments are seldom and have shown more or less success, in particular if one looks for quantitative agreement. Thus, to make them useful in particular for the engineering sciences, comparison with experiments have to be done in a deeper and more extensive way.

It is planed in the next years during the ANR Programme SYSCOMM (ANR-09-SYSC-006 Mechanics and Statistical Physics of Rupture in Brittle Heterogeneous Materials) that supported this work.

\section{Acknowledgements}

The support of the ANR Programme SYSCOMM (ANR-09-SYSC-006) is gratefully acknowledged.

I would also like to greatly thank E. Bouchaud, J.R. Rice, L. Vanel, J.B. Leblond, M. Adda-Bedia, J.J. Marigo, S. Roux, J. Schmittbuhl who kindly accepted to participate to the examiners board of my "Habilitation à diriger des Recherches" exam and who encouraged me to publish this work. A particular thank goes to D. Bonamy for its careful review. 


\section{References}

Adda-Bedia, M., Mahadevan, L., 2006. Crack-front instability in a confined elastic film. Proceedings of the royal society A-Mathematical physical and engineering sciences 462 (2075), 3233 - 3251.

Amestoy, M., Leblond, J.-B., 1992. Crack Paths in Plane Situations - II. Detailed Form of the Expansion of the Stress Intensity Factors. International Journal of Solids and Structures 29, 465-501.

Anderson, T. L., 1991. Fracture Mechanics: Fundamentals and Applications. CRC Press, Inc., Boca Raton, USA.

Atkinson, B. K., 1987. Fracture mechanics of rock. Academic Press geology series.

Aydin, A., Pollard, D. D., 1988. Progress in understanding jointing over the past century. Geological Society of America Bulletin 100, 1181-1204.

Barabási, A.-L., Stanley, H. E., 1995. Fractal concepts in surface growth.

Bazant, Z. P., Cedolin, L., 2003. Stability of Structures: Elastic, Inelastic, Fracture, and Damage Theories, new ed edition (february 14, 2003) Edition. Dover Publications.

Benallal, A., Marigo, J.-J., 2007. Bifurcation and stability issues in gradient theories with softening. Modelling and Simulation in Materials Science and Engineering 15, S283-S295.

Bercial-Velez, J., Antipov, Y., Movchan, A., May 2005. High-order asymptotics and perturbation problems for $3 \mathrm{~d}$ interfacial cracks. Journal of the Mechanics and Physics of Solids 53 (5), 1128-1162.

Bonamy, D., 2009. Intermittency and roughening in the failure of brittle heterogeneous materials. Journal of Physics D: Applied Physics 42 (21), 214014 (21pp).

Bouchbinder, E., Livne, A., Fineberg, J., 03 2010. Weakly nonlinear fracture mechanics: experiments and theory. International Journal of Fracture 162 (1), 3-20. 
Bourdin, B., Francfort, G., Marigo, J.-J., 2008. The variational approach to fracture. Journal of elasticity 91 (1), 5 - 148.

Bourdin, B., Francfort, G. A., Marigo, J. J., 2000. Numerical experiments in revisited brittle fracture. Journal of the Mechanics and Physics of Solids 48 (4), 797-826.

Bower, A. F., Ortiz, M., 1990. Solution of three-dimensional crack problems by a finite perturbation method. Journal of the Mechanics and Physics of Solids 38 (4), 443-480.

Bower, A. F., Ortiz, M., 1991. A three-dimensional analysis of crack trapping and bridging by tough particles. Journal of the Mechanics and Physics of Solids 39 (6), 815-858.

Bower, A. F., Ortiz, M., 1993. An analysis of crack trapping by residual stresses in brittle solids. Transactions of the ASME. Journal of Applied Mechanics 60 (1), 175-82.

Broberg, K. B., 1999. Cracks and fracture. Academic Press.

Budiansky, B., Amazigo, J. C., Evans, A. G., 1988. Small-scale crack bridging and the fracture toughness of particulate-reinforced ceramics. Journal of the Mechanics and Physics of Solids 36 (2), $167-187$.

Bueckner, H. F., 1970. A novel principle for the computation of stress intensity factors. Zeitschrift fur Angewandte Mathematik und Mechanik 50 (9), $529-546$.

Bueckner, H. F., 1987. Weight functions and fundamental fields for the penny-shaped and the half-plane crack in three-space. International Journal of Solids and Structures 23 (1), 57-93.

Caginalp, G., Fife, P., 1986. Phase field methods for interfacial boundaries. Physical Review B 33, 7792-7794.

Chambolle, A., Francfort, G., Marigo, J.-J., 2009. When and how do cracks propagate? Journal of the Mechanics and Physics of Solids 57 (9), 1614 1622. 
Ciavarella, M., Paggi, M., Carpinteri, A., 2008. One, no one, and one hundred thousand crack propagation laws: A generalized barenblatt and botvina dimensional analysis approach to fatigue crack growth. Journal of the Mechanics and Physics of Solids 56 (12), 3416 - 3432.

Collins, J., Levine, H., 1985. Diffuse interface model of diffusion-limited crystal-growth. Physical review B 31 (9), 6119-6122.

Cooke, M. L., Pollard, D. D., 1996. Fracture propagation paths under mixed mode loading within rectangular blocks of polymethyl methacrylate. Journal of Geophysical Research 101 (B2), 3387-3400.

Corson, F., Adda-Bedia, M., Henry, H., Katzav, E., 2009. Thermal fracture as a framework for quasi-static crack propagation. International Journal of Fracture 158 (1), 1-14.

Cotterell, B., Rice, J. R., 1980. Slightly curved or kinked cracks. International Journal of Fracture 16 (2), 155-169.

Daguier, P., Bouchaud, E., Lapasset, G., 1995. Roughness of a crack front pinned by microstructural obstacles. EPL (Europhysics Letters) 31 (7), 367-372.

Dalmas, D., Barthel, E., Vandembroucq, D., 2009. Crack front pinning by design in planar heterogeneous interfaces. Journal of the Mechanics and Physics of Solids 57 (3), $446-457$.

Delaplace, A., Schmittbuhl, J., Mȧløy, K. J., 1999. High resolution description of a crack front in a heterogeneous plexiglas block. Physical Review E (Statistical Physics, Plasmas, Fluids, and Related Interdisciplinary Topics) 60 (2), 1337-43.

Doquet, V., Bertolino, G., 2008. Local approach to fatigue cracks bifurcation. International journal of fatigue 30 (5), 942-950.

Drazin, P. G., 1992. Nonlinear systems. Press Syndicate of the University of Cambridge, Cambridge.

Erdogan, G., Paris, P., 1963. A critical analysis of crack propagation laws. ASME J. Basic Engng Trans. 85, 528-534. 
Erdogan, G., Sih, G. C., 1963. On the crack extension in plates under plane loading and transverse shear. ASME J. Basic Engng 85, 519-527.

Family, F., Vicsek, T., 1985. Scaling of the active zone in the eden process on percolation networks and the ballistic deposition model. Journal of Physics A: Mathematical and General 18 (2), L75.

Favier, E., Lazarus, V., Leblond, J.-B., 2006a. Coplanar propagation paths of $3 \mathrm{D}$ cracks in infinite bodies loaded in shear. International Journal of Solids and Structures 43 (7-8), 2091-2109.

Favier, E., Lazarus, V., Leblond, J.-B., 2006b. Statistics of the deformation of the front of a tunnel-crack propagating in some inhomogeneous medium. Journal of the Mechanics and Physics of Solids 54 (7), 1449-1478.

Fineberg, J., Marder, M., 1999. Instability in dynamic fracture. Physics Reports-Rev. Sec. Phys. Lett. 313 (1-2), 2-108.

Fisher, D. S., Dahmen, K., Ramanathan, S., Ben-Zion, Y., 1997. Statistics of earthquakes in simple models of heterogeneous faults. Physical Review Letters 78 (25), 4885-8.

Fleck, N., Kang, K., Ashby, M., 1994. Overview no. 112: The cyclic properties of engineering materials. Acta Metallurgica et Materialia 42 (2), 365 - 381.

Francfort, G. A., Marigo, J. J., 1998. Revisiting brittle fracture as an energy minimization problem. Journal of the Mechanics and Physics of Solids 46, 1319-1342.

Freund, L., 1972a. Crack propagation in an elastic solid subjected to general loading-i. constant rate of extension. Journal of the Mechanics and Physics of Solids 20 (3), $129-140$.

Freund, L., 1972b. Crack propagation in an elastic solid subjected to general loading-ii. non-uniform rate of extension. Journal of the Mechanics and Physics of Solids 20 (3), $141-152$.

Freund, L., 1973. Crack propagation in an elastic solid subjected to general loading-iii. stress wave loading. Journal of the Mechanics and Physics of Solids $21(2), 47-61$. 
Freund, L. B., 1974. Crack propagation in an elastic solid subjected to general loading-iv. obliquely incident stress pulse. Journal of the Mechanics and Physics of Solids 22 (3), $137-146$.

Freund, L. B., 2000. Substrate curvature due to thin film mismatch strain in the nonlinear deformation range. Journal of the Mechanics and Physics of Solids 48 (6-7), 1159-1174.

Gao, H., 1988. Nearly circular shear mode cracks. International Journal of Solids and Structures 24 (2), 177-193.

Gao, H., Rice, J. R., 1986. Shear stress intensity factors for planar crack with slightly curved front. ASME Journal of Applied Mechanics 53 (4), 774-778.

Gao, H., Rice, J. R., 1987a. Nearly circular connections of elastic half spaces. ASME Journal of Applied Mechanics 54 (4), 627-634.

Gao, H., Rice, J. R., 1987b. Somewhat circular tensile cracks. International Journal of Fracture 33 (3), 155-174.

Gao, H., Rice, J. R., 1989. A first-order perturbation analysis of crack trapping by arrays of obstacles. Transactions of the ASME 56, 828-836.

Gao, H., Rice, J. R., Lee, J., 1991. Penetration of a quasi-statically slipping crack into a seismogenic zone of heterogeneous fracture resistance. Journal of Geophysical Research 96 (B13), 21535-21548.

Gauthier, G., Lazarus, V., Pauchard, L., 2010. Shrinkage star-shaped cracks: Explaining the transition from 90 degrees to 120 degrees. EPL 89, 26002.

Ghatak, A., Chaudhury, M. K., 2003. Adhesion-induced instability patterns in thin confined elastic film. Langmuir 19 (7), 2621-2631.

Goehring, L., Mahadevan, L., Morris, S. W., 2009. Nonequilibrium scale selection mechanism for columnar jointing. Proceedings of the National Academy of Sciences 106 (2), 387-392.

Goldstein, R. V., Salganik, R. L., 1974. Brittle fracture of solids with arbitrary cracks. International Journal of Fracture 10, 507-523. 
Griffith, A. A., 1920. The phenomena of rupture and flow in solids. Philosophical Transactions of the Royal Society of London 221, 163-198.

Grob, M., Schmittbuhl, J., Toussaint, R., Rivera, L., Santucci, S., Maloy, K. J., JUL 2009. Quake Catalogs from an Optical Monitoring of an Interfacial Crack Propagation. Pure and applied geophysics 166 (5-7), 777-799.

Hakim, V., Karma, A., 2009. Laws of crack motion and phase-field models of fracture. Journal of the Mechanics and Physics of Solids 57 (2), $342-$ 368 .

Hansen, A., Schmittbuhl, J., 2003. Origin of the universal roughness exponent of brittle fracture surfaces: stress-weighted percolation in the damage zone. Physical Review Letters 90 (4), 045504/1-4.

Henry, H., Levine, H., 2004. Dynamic instabilities of fracture under biaxial strain using a phase field model. Physical Review Letters 93 (10), 105504.

Hull, D., 1993. Tilting cracks: the evolution of fracture surface topology in brittle solids. International Journal of Fracture 62 (2), 119-138.

Irwin, G. R., 1957. Analysis of stresses and strains near the end of a crack traversing a plate. Journal of Applied Mechanics 24, 361-364.

Irwin, G. R., 1958. Fracture. Hand. der Physik. Vol. IV. Springer, Berlin.

Kardar, M., 1998. Nonequilibrium dynamics of interfaces and lines. Physics Reports-Rev. Sec. Phys. Lett. 301 (1-3), 85-112.

Karma, A., Kessler, D. A., Levine, H., 2001. Phase-field model of mode III dynamic fracture. Physical Review Letters 87, 045501.

Karma, A., Leblond, J. B., Pons, A. J., Lazarus, V., 2011. Instability of crack propagation with superposition of antiplane shear and tension (In preparation).

Kassir, M. K., Sih, G., 1975. Three Dimensional Crack Problems. Nordhoff International Publishing, Leyden, The Netherlands.

Katzav, E., Adda-Bedia, M., 2006. Roughness of tensile crack fronts in heterogenous materials. Europhysics Letters 76 (3), 450-456. 
Kostrov, B., 1975. On the crack propagation with variable velocity. International Journal of Fracture 11, 47-56.

Lancioni, G., Royer-Carfagni, G., APR 2009. The Variational Approach to Fracture Mechanics. A Practical Application to the French Panth,on in Paris. Journal of elasticity 95 (1-2), 1-30.

Lange, F. F., 1970. The interaction of a crack front with a second-phase dispersion. Philosophical Magazine 22 (179).

Lazarus, V., 2003. Brittle fracture and fatigue propagation paths of 3D plane cracks under uniform remote tensile loading. International Journal of Fracture 122 (1-2), 23-46.

Lazarus, V., Buchholz, F.-G., Fulland, M., Wiebesiek, J., 2008. Comparison of predictions by mode II or mode III criteria on crack front twisting in three or four point bending experiments. International Journal of Fracture 153, 141-151.

Lazarus, V., Gauthier, G., Pauchard, L., Maurini, C., Valdivia, C., July 2009. Basalt columns and crack formation during directional drying of colloidal suspensions in capillary tubes. In: 12th International Conference on Fracture (ICF12). Ottawa (Canada).

Lazarus, V., Leblond, J., Karma, A., 2011a. Segmentation and coarsening in presence of mode 3: experimental study (In preparation).

Lazarus, V., Leblond, J.-B., 1998a. Crack paths under mixed mode (I+III) or (I+II+III) loadings. Comptes Rendus de l'Académie des Sciences, Série II (Mécanique, Physique, Astronomie) 326 (3), 171-177.

Lazarus, V., Leblond, J.-B., 1998b. Three-dimensional crack-face weight functions for the semi-infinite interface crack. I. Variation of the stress intensity factors due to some small perturbation of the crack front. Journal of the Mechanics and Physics of Solids 46 (3), 489-511.

Lazarus, V., Leblond, J.-B., 2002a. Crack front stability for a tunnel-crack propagating along its plane in mode $2+3$. Comptes Rendus de l'Académie des Sciences Paris, Serie II (Mecanique, Physique, Astronomie) 330 (6), 437-443. 
Lazarus, V., Leblond, J.-B., 2002b. In-plane perturbation of the tunnel-crack under shear loading. I: Bifurcation and stability of the straight configuration of the front. International Journal of Solids and Structures 39 (17), 4421-4436.

Lazarus, V., Leblond, J.-B., 2002c. In-plane perturbation of the tunnel-crack under shear loading. II: determination of the fundamental kernel. International Journal of Solids and Structures 39 (17), 4437-4455.

Lazarus, V., Leblond, J.-B., Mouchrif, S.-E., 2001a. Crack front rotation and segmentation in mixed mode I+III or I+II+III - part I: Calculation of Stress Intensity Factor. Journal of the Mechanics and Physics of Solids 49 (7), 1399-1420.

Lazarus, V., Leblond, J.-B., Mouchrif, S.-E., 2001b. Crack front rotation and segmentation in mixed mode I+III or I+II+III - part II: Comparison with experiments. Journal of the Mechanics and Physics of Solids 49 (7), 1421-1443.

Lazarus, V., Maurini, C., Gauthier, G., Bourdin, B., 2011b. Crack patterns in directional drying: experiments and results of numerical energy minimization (In preparation).

Leblond, J., Karma, A., Lazarus, V., 2011. Theoretical analysis of crack front instability in mode I+III. Journal of the mechanics and physics of solides (Submitted).

Leblond, J.-B., 1989. Crack Paths in Plane Situations - I. General Form of the Expansion of the Stress Intensity Factors. International Journal of Solids and Structures 25, 1311-1325.

Leblond, J.-B., 1999. Crack paths in three-dimensional elastic solids. i: twoterm expansion of the stress intensity factors-application to crack path stability in hydraulic fracturing. International Journal of Solids and Structures $36(1), 79-103$.

Leblond, J.-B., Lazarus, V., Mouchrif, S.-E., 1999. Crack paths in threedimensional elastic solids. II. Three-term expansion of the stress intensity factors - Applications and perspectives. International Journal of Solids and Structures 36 (1), 105-142. 
Leblond, J.-B., Leguillon, D., 1999. Asymptotic behavior of stress intensity factors near an angular point of a crack front. European Journal of Mechanics, A/Solids 18 (1), 135-145.

Leblond, J.-B., Mouchrif, S.-E., Perrin, G., 1996. The tensile tunnel-crack with a slightly wavy front. International Journal of Solids and Structures 33 (14), 1995-2022.

Legrand, L., Leblond, J. B., 2010a. Evolution of the shape of the fronts of a pair of semi-infinite cracks during their coplanar coalescence. ZAMM 90 (10-11), 821-836.

Legrand, L., Leblond, J.-B., 2010b. In-plane perturbation of a system of two coplanar slit-cracks - II: Case of a large distance between the outer crack fronts. International Journal of Solids and Structures 47, 3504-3512.

Lin, B., Mear, M., Ravi-Chandar, K., 2010. Criterion for initiation of cracks under mixed-mode I + III loading. International Journal of Fracture 165, $175-188$.

Liu, Y., Rice, J., 2005. Aseismic slip transients emerge spontaneously in three-dimensional rate and state modeling of subduction earthquake sequences. Journal of geophysical research-Solid earth 110 (B8).

Maloy, K., Schmittbuhl, J., 2001. Dynamical event during slow crack propagation. Physical Review Letters 87 (10), 105502/1-4.

Meade, K. P., Keer, L. M., 1984. On the problem of a pair of point forces applied to the faces of a semi-infinite plane crack. Journal of Elasticity $14(1), 3-14$.

Morrissey, J., Rice, J., 1998. Crack front waves. Journal of the mechanics and physics of solids 46 (3), $467-487$.

Morrissey, J., Rice, J., 2000. Perturbative simulations of crack front waves. Journal of the mechanics and physics of solids 48 (6-7), $1229-1251$.

Movchan, A. B., Gao, H., Willis, J. R., 1998. On perturbations of plane cracks. International Journal of Solids and Structures 35 (26-27), 34193453 . 
Mower, T., Argon, A., 1995. Experimental investigations of crack trapping in brittle heterogeneous solids. Mechanics of Materials 19 (4), 343-64.

Nguyen, Q.-S., 2000. Stability and nonlinear solid mechanics. John Wiley.

Obrezanova, O., Movchan, A., Willis, J., 2002a. Dynamic stability of a propagating crack. Journal of the mechanics and physics of solids 50 (12), 26372668 .

Obrezanova, O., Movchan, A., Willis, J., 2002b. Stability of an advancing crack to small perturbation of its path. Journal of the Mechanics and Physics of Solids 50 (1), 57-80.

Paris, P., Gomez, M., Anderson, W., 1961. A rational analytic theory of fatigue. Trends Eng. 13, 94.

Perrin, G., Rice, J. R., 1994. Disordering of a dynamic planar crack front in a model elastic medium of randomly variable toughness. Journal of the Mechanics and Physics of Solids 42 (6), 1047-1064.

Piccolroaz, A., Mishuris, G., Movchan, A. B., 2007. Evaluation of the Lazarus-Leblond constants in the asymptotic model of the interfacial wavy crack. Journal of the Mechanics and Physics of Solids 55 (8), 1575-1600.

Pindra, N., Lazarus, V., Leblond, J.-B., 2008. The deformation of the front of a 3d interface crack propagating quasistatically in a medium with random fracture properties. Journal of the Mechanics and Physics of Solids 56 (4), 1269-1295.

Pindra, N., Lazarus, V., Leblond, J.-B., 2010a. Geometrical disorder of the fronts of a tunnel-crack propagating in shear in some heterogeneous medium. Journal of the Mechanics and Physics of Solids 58, 281-299.

Pindra, N., Lazarus, V., Leblond, J.-B., 2010b. In-plane perturbation of a system of two coplanar slit-cracks - I: Case of arbitrarily spaced crack fronts. International Journal of Solids and Structures 47, 3489-3503.

Pons, A. J., Karma, A., 2010. Helical crack-front instability in mixed-mode fracture. Nature 464, 85-89.

Qian, J., Fatemi, A., 1996. Mixed mode fatigue crack growth: a literature survey. Engineering Fracture Mechanics 55 (6), 969. 
Ramanathan, S., Fisher, D. S., 1997. Dynamics and instabilities of planar tensile cracks in heterogeneous media. Physical Review Letters 79 (5), $877-80$.

Ravi-Chandar, K., 1998. Dynamic fracture of nominally brittle materials. International journal of fracture 90 (1-2), 83-102.

Rice, J., Ben-Zion, Y., Kim, K., 1994. 3-Dimensional perturbation solution for a dynamic planar crack moving unsteadily in a model elastic solid. Journal of the mechanics and physics of solids 42 (5), 813-843.

Rice, J. R., 1972. Some remarks on elastic crack-tip stress fields. International Journal of Solids and Structures 8 (6), 751-758.

Rice, J. R., 1985. First-order variation in elastic fields due to variation in location of a planar crack front. ASME Journal of Applied Mechanics 52 (3), $571-579$.

Rice, J. R., 1988. Crack fronts trapped by arrays of obstacles: Solutions based on linear perturbation theory. In: Rosakis, A. J., Ravi-Chandar, K., Rajapakse, Y. (Eds.), Analytical, Numerical and Experimental Aspects of Three Dimensional Fracture Processes. Vol. 91. ASME Applied Mechanics Division, American Society of Mechanical Engineers, New York, pp. 175184.

Rice, J. R., 1989. Weight function theory for three-dimensional elastic crack analysis. In: Wei, R. P., Gangloff, R. P. (Eds.), Fracture Mechanics : Perspectives and Directions (Twentieth Symposium). American Society for Testing and Materials STP 1020, Philadelphia, USA, pp. 29-57.

Rosso, A., Krauth, W., 2002. Roughness at the depinning threshold for a long-range elastic string. Physical review E 65 (2), 025101.

Roux, S., Vandembroucq, D., Hild, F., 2003. Effective toughness of heterogeneous brittle materials. European Journal of Mechanics - A/Solids 22 (5), 743-749.

Santucci, S., Grob, M., R.Toussaint, Schmittbuhl, J., Hansen, A., Måløy, K. J., July 2009. Crackling dynamics during the failure of heterogeneous material: Optical and acoustic tracking of slow interfacial crack growth. In: 12th International Conference on Fracture (ICF12). Ottawa (Canada). 
Schmittbuhl, J., Delaplace, A., Màløy, K. J., Perfettini, H., Vilotte, J. P., 2003a. Slow crack propagation and slip correlations. Pure and applied geophysics 160 (5-6), 961-976.

Schmittbuhl, J., Hansen, A., Batrouni, G., 2003b. Roughness of interfacial crack fronts: stress-weighted percolation in the damage zone. Physical Review Letters 90 (4), 045505/1-4.

Schmittbuhl, J., Màløy, K. J., 1997. Direct observation of a self-affine crack propagation. Physical Review Letters 78 (20), 3888-91.

Schmittbuhl, J., Roux, S., Vilotte, J. P., Maloy, K.-J., 1995. Interfacial crack pinning: effect of nonlocal interactions. Physical Review Letters 74 (10), 1787-1790.

Schmittbuhl, J., Vilotte, J.-P., 1999. Interfacial crack front wandering: influence of quenched noise correlations. Physica A 270 (1-2), 42-56.

Sharon, E., Fineberg, J., JAN 28 1999. Confirming the continuum theory of dynamic brittle fracture for fast cracks. NATURE 397 (6717), 333-335.

Stallybrass, M., 1981. On the concentrated loading of certain elastic halfspace problems and related external crack problems. A new approach. International Journal of Engineering Science 19 (8), 1123-1144.

Tada, H., Paris, P. C., Irwin, G. R., 1973. The Stress Analysis of Cracks Handbook. Del Research Corporation, Hellertown, USA.

Vieira, A. P., Jose S. Andrade, J., Herrmann, H. J., 2008. Subcritical crack growth: The microscopic origin of Paris' law. Physical Review Letters 100 (19), 195503, origine physic de la loi de Paris.

Willis, J., Movchan, A., 1997. Three-dimensional dynamic perturbation of a propagating crack. Journal of the mechanics and physics of solids 45 (4), 591-610.

Willis, J. R., Movchan, A. B., 1995. Dynamic weight-functions for a moving crack. 1. mode-I loading. J. Mech. Phys. Solids 43 (3), 319-341.

Woolfries, S., Willis, J. R., 1999. Perturbation of a dynamic planar crack moving in a model elastic solid. Journal of the Mechanics and Physics of Solids 47 (8), 1633-1661. 